\title{
What is the temperature structure in the giant HII region NGC 588?^
}

\author{
L. Jamet ${ }^{1,2}$, G. Stasińska ${ }^{1}$, E. Pérez ${ }^{2}$, R. M. González Delgado ${ }^{2}$, and J. M. Vílchez ${ }^{2}$ \\ 1 LUTH, Observatoire de Meudon, 5 place Jules Janssen, 92195 Meudon Cedex, France \\ 2 Instituto de Astrofísica de Andalucía (CSIC), Apartado 3004, 18080 Granada, Spain \\ e-mail: luc@iaa.es
}

Received 22 November 2004 / Accepted 29 July 2005

ABSTRACT

We present the results of an exhaustive study of the ionized gas in NGC 588, a giant HII region in the nearby spiral galaxy M 33 . This analysis uses a high number of diagnostics in the optical and infrared ranges. Four temperature diagnostics obtained with optical lines agree with a gas temperature of $11000 \mathrm{~K}$, while the [OIII] $\lambda 5007 / \lambda 88 \mu \mathrm{m}$ ratio yields a much lower temperature of $\approx 8000 \mathrm{~K}$. This discrepancy suggests the presence of large temperature inhomogeneities in the nebula. We investigated the cause of this discrepancy by constructing photoionization models of increasing complexity. In particular, we used the constraints from the $\mathrm{H} \alpha$ and $\mathrm{H} \beta$ surface brightness distributions and state-of-theart models of the stellar ionizing spectrum. None of the successive attempts was able to reproduce the discrepancy between the temperature diagnostics, so the thermal balance of NGC 588 remains unexplained. We give an estimate of the effect of this failure on the $\mathrm{O} / \mathrm{H}$ and $\mathrm{Ne} / \mathrm{O}$ estimates and show that $\mathrm{O} / \mathrm{H}$ is known to within \pm 0.2 dex.

Key words. ISM: abundances - ISM: HII regions - ISM: individual objects: NGC 588 - galaxies: individual: M 33

\section{Introduction}

Giant HII regions (GHRs) are the most popular tracers of elemental abundances in distant galaxies, since they are easy to observe and, in principle, easy to analyze. Abundances can be obtained directly from the intensities of emission lines, without having to go through a detailed model analysis to extract the information (e.g., Stasińska 2004). However, for metal-rich HII regions - say, with an oxygen abundance larger than half that of the Sun - the methods for abundance derivation are statistical and rely on previous calibrations based on photoionization models. The large number of calibrations that have been published, leading to significantly different abundances (e.g., Kennicutt et al. 2003), show that the interpretation of emission lines in HII regions may not be as easy as one would like. In the case of metallicities significantly below solar, abundance determination is considered more reliable, since the optical emission lines provide a direct measure of the gas temperature, which allows one to compute the elemental abundances directly from the observed line intensities. However, as shown by Peimbert (1967), if the temperature in HII regions is not uniform but instead presents spatial fluctuations, the derived abundances will be biased. The reality of the presence of these temperature fluctuations and their cause are still a matter of debate (e.g., Esteban 2002).

* Appendices A and B are only available in electronic form at http://www. edpsciences.org
Comprehensive analysis of nearby giant HII regions are necessary to check our understanding of the thermal structure of these objects and to validate empirical methods for abundance determinations. There have already been detailed photoionization studies of some giant HII regions (García-Vargas et al. 1997; Stasińska \& Schaerer 1999; Luridiana et al. 1999, 2003; González Delgado \& Pérez 2000; Luridiana \& Peimbert 2001; Relaño et al. 2002). In most cases, the models were not able to reproduce the observed temperature indicators correctly. However, this was not the main goal of most of those studies, and the degree of sophistication of the models was perhaps insufficient for that purpose.

In the present paper, we propose a comprehensive analysis of NGC 588, a giant HII region located on the outskirts of the nearby spiral galaxy M 33, with the aim of understanding its temperature structure. We gathered a large set of spectroscopic and imaging data in various wavelength ranges. From this set of data, we were able to give a full description of the ionizing stellar population, based on a star-by-star analysis (Jamet et al. 2004). We now use the ionizing radiation field from this population together with information on the nebular morphology given by narrow band images to construct photoionization models. Constraints are provided by the strengths of optical and infrared emission lines. The availability of infrared data is particularly important, since they enlarge the number of possible spectral diagnostics.

The paper is organized as follows. In Sect. 2 we describe the observational data and their processing. In Sect. 3 we present 
Table 1. Journal of observations.

\begin{tabular}{|c|c|c|}
\hline \multicolumn{3}{|l|}{ Spectroscopy } \\
\hline \multicolumn{3}{|c|}{ CAHA $\left(\mathrm{PA}=121^{\circ}\right.$, width $\left.=1.2^{\prime \prime}, 1998-08-27 / 28\right)$} \\
\hline range: $\lambda(\AA)$ & $\Delta \lambda(\AA /$ pixel $)$ & exposure (s) \\
\hline B: $3595-5225$ & 0.81 & $4 \times 1800$ \\
\hline R1: 5505-7695 & 1.08 & $2 \times 1800$ \\
\hline R2: 7605-9805 & 1.08 & $2 \times 1800$ \\
\hline \multicolumn{3}{|c|}{ ISO/SWS $\left(\mathrm{PA}=-29^{\circ}\right.$, data set $\left.81601775,1998-02-08\right)$} \\
\hline line & range $(\mu \mathrm{m})$ & aperture (") \\
\hline$[\mathrm{SIV}] \lambda 11 \mu \mathrm{m}$ & $10.45-10.55$ & $14 \times 20$ \\
\hline [NeIII] $\lambda 16 \mu \mathrm{m}$ & $15.46-15.64$ & $14 \times 27$ \\
\hline$[\mathrm{SIII}] \lambda 19 \mu \mathrm{m}$ & $18.61-18.77$ & $14 \times 27$ \\
\hline [OIV] $\lambda 26 \mu \mathrm{m}$ & $25.80-26.08$ & $14 \times 27$ \\
\hline$[\mathrm{SIII}] \lambda 34 \mu \mathrm{m}$ & $33.17-33.75$ & $20 \times 33$ \\
\hline$[\mathrm{NeIII}] \lambda 36 \mu \mathrm{m}$ & $35.70-36.26$ & $20 \times 33$ \\
\hline \multicolumn{3}{|l|}{ ISO/LWS } \\
\hline Data set & Spectrometer & Exposure (s) \\
\hline 59901081 & LWS01 & 1478 \\
\hline 80800268 & LWS02 & 810 \\
\hline 81601776 & LWS01 & 1402 \\
\hline \multicolumn{3}{|l|}{ Imaging } \\
\hline \multicolumn{3}{|c|}{ JKT (1990-08-20/21) } \\
\hline Band & Filter & Exposure (s) \\
\hline $\mathrm{H} \beta$ & $4861 / 54$ & $2 \times 1800$ \\
\hline $\mathrm{H} \alpha$ & $6563 / 53$ & 1800 \\
\hline $\mathrm{H} \alpha$ continuum & $6834 / 51$ & 1300 \\
\hline [OIII] $\lambda 5007$ & $5007 / 51$ & 1800 \\
\hline
\end{tabular}

empirical diagnostics of the density, temperature, and chemical composition. In Sect. 4 we give details on the modelfitting strategy that we adopted. Starting from very simple models (Sect. 5), we gradually increased the degree of sophistication in order to match the observations as closely as possible (Sects. 6-7). We then examine the effects of energy sources other than the ionizing flux of the cluster (Sect. 8). In Sect. 9, we evaluate the impact of the unknowns of the thermal structure of the nebula on the determination of the $\mathrm{O} / \mathrm{H}$ and $\mathrm{Ne} / \mathrm{O}$ abundance ratios. Finally, we present our conclusions in Sect. 10.

\section{Observational data and reduction}

We acquired ground-based optical spectra of NGC 588 and retrieved a series of data available for this object in the archives of the Infrared Space Observatory (ISO) and of the Isaac Newton Group (ING). The main properties of the data with which we realized measurements are summarized in Table 1. Figure 1 shows the nebula in $\mathrm{H} \alpha$ (see Sect. 2.2) on which the spectroscopic slits were superimposed.

\subsection{Optical CAHA spectra}

We obtained a set of 3 spectra on 27 August 1998 with the $3.5 \mathrm{~m}$ telescope of the Centro Astronómico Hispano Alemán (CAHA, Calar Alto, Almería, Spain). These observations were

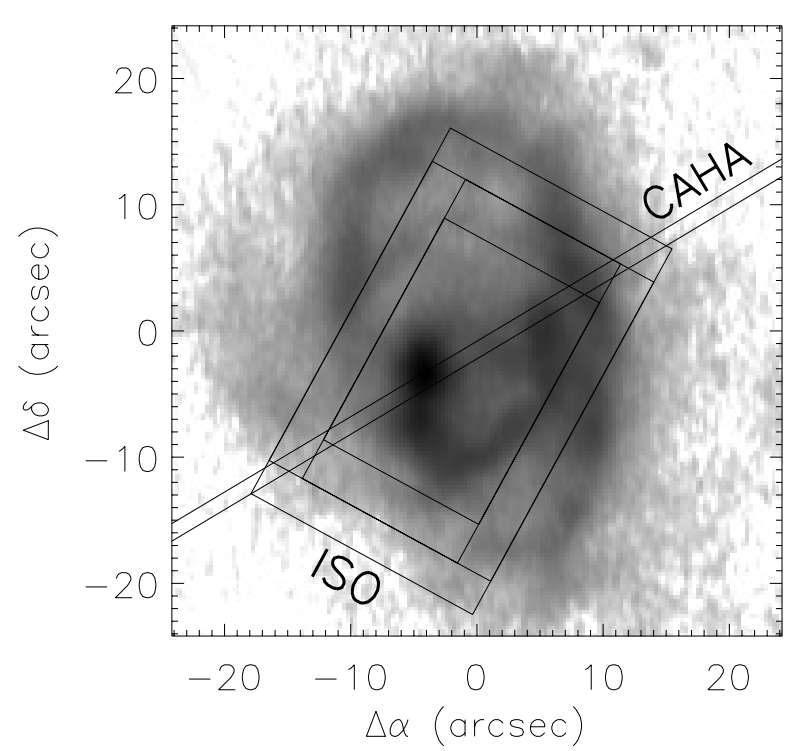

Fig. 1. H $\alpha$ image with the spectroscopic slits overplotted. North is up, and east is on the left. The image display scale is logarithmic.

acquired with the dual beam TWIN spectrograph, equipped with a beam splitter centered on $5500 \AA$ which sends the signal through two arms ("red" and "blue"). The spectrum observed with each arm was collected with an SITe-CCD of $2000 \times 80015 \mu \mathrm{m}$ pixels. We took four exposures in the range $3595-5225 \AA$ (range B) with the blue arm, two in the range 5505-7695 $\AA$ (range R1), and two in the range 7605-9805 (range R2) with the red arm; each of these exposures was $1800 \mathrm{~s}$ long. The two gratings used for the observations, T7 (blue arm) and T4 (red arm), give dispersions of 0.81 and $1.08 \AA /$ pix, respectively. The signal was acquired through a $240 \times 1.2^{\prime \prime}$ slit oriented at $\mathrm{PA}=121^{\circ}$ with a resolution of $\sim 2 \AA$ along the total spectral range, as measured from the $F W H M$ of the lines of the sky background.

\subsubsection{Reduction}

We reduced the bidimensional spectra following the standard procedure, mainly making use of the IRAF $^{1}$ package noao. twodspec. longslit.

The flat-field correction was made with two light sources: an internal tungsten lamp for removal of the effect of pixel-topixel sensitivity variations and the twilight sky for correction for instrumental vignetting. Though the tungsten lamp spectra were heavily affected by fringing effects, the overal sensitivity flattening was satisfactory; we estimated the residuals to be $\sim 1 \%$.

The wavelength calibration was performed from spectra of a helium-argon arc lamp, typically giving 20 useful lines per frame. Several of these spectra were obtained throughout the night in order to account for instrumental drifts.

${ }^{1}$ IRAF is distributed by the National Optical Astronomy Observatory, which is operated by the Association of Universities for Research in Astronomy, Inc., under cooperative agreement with the National Science Foundation. 
No data was available to calibrate the frames with respect to the positions along the slit, and the inspection of several spectra of stars showed $\sim 2$ pixel $\left(\approx 1^{\prime \prime}\right)$ distorsions around horizontal lines of pixels. Since we were mainly interested in integrated line fluxes, we ignored this issue, except for the hydrogen lines used for dereddening (see Sect. 2.1.2).

We carefully combined the individual exposures in each range (B, R1, R2). We first performed slight shifts of these frames along the slit axis, to match the profiles of the lines, since the instrument centering on the object was subject to small variations between the different exposures. The differences between the coregistered frames showed no detectable residuals other than cosmic rays, except for some of the brightest lines $(\mathrm{H} \alpha, \mathrm{H} \beta$, and [OIII] 15007$)$ in a $\sim 4^{\prime \prime}$ zone around the maximum nebular emission. However, these discrepancies were small and resulted in flux errors less than $\sim 3 \%$ in this zone. Since this error is small and concerns only a small zone of the slit, we neglected it.

The combined frames were then flux-calibrated. To this aim, the photometric response of the instrument was measured with three standard stars, BD+28 4211, G191-B2B and GD71, observed with a $3.6^{\prime \prime}$ wide slit and through airmasses similar to the one of the observations of NGC 588 (less than 1.2). We excluded the stellar lines from the measurement points, because of the difference of spectral resolution between our observations and the reference spectra. The atmospheric extinction function we adopted is the average one of the Observatorio de Roque de los Muchachos (La Palma, Spain), located at an altitude very similar to the one at CAHA. In each of the three spectral ranges, we found the response measures, as obtained with the different available spectra, to show the same chromatic trends, but constant discrepancies of $\sim 0.1 \mathrm{mag}$. We also found oscillations of amplitude $\approx 3 \%$ on scales of 50-200 $\AA$, which we were unable to fit. Because of the drop in the instrumental response, the $\lambda<4000 \AA$ range is more uncertain at $\sim 15 \%$ with respect to the overall range $\mathrm{B}$. Furthermore, in our data, a series of wide regions in the range $\mathrm{R} 1$ and, above all, $\mathrm{R} 2$ suffer severe extinction by telluric $\mathrm{O}_{2}$ and $\mathrm{H}_{2} \mathrm{O}$ unresolved molecular bands. Such extinction tended to cause numerical instabilities when fitting smooth functions on the measured photometric response, so we decided to correct them, even coarsely, to avoid these instabilities. For this, we synthesized the $\mathrm{O}_{2}$ and $\mathrm{H}_{2} \mathrm{O}$ molecular bands, starting from individual lines as catalogued in the solar atlases of Moore et al. (1966) and Mohler (1950). In both atlases, only wavelength centers $\lambda_{i}$ and line strengths $W_{i}$ are available, and we decided to model the bands as functions of magnitude loss of the form $F(\lambda ; \mathrm{X})=N(\mathrm{X}) \sum_{i} W_{i} H\left(\lambda-\lambda_{i}\right)$, with $\mathrm{X}=\mathrm{O}_{2}$ or $\mathrm{H}_{2} \mathrm{O}, N(\mathrm{X})$, the relative column density of $\mathrm{X}$ on the line of sight, and $H(\lambda)$, the Gaussian spectral PSF of the spectrograph. We then corrected the observed spectra of the standard stars for these extinction functions, using values of $N\left(\mathrm{O}_{2}\right)$ and $N\left(\mathrm{H}_{2} \mathrm{O}\right)$ that smoothed the corrected spectra best, and we computed the instrumental response in the ranges $\mathrm{R} 1$ and R2 with them.

We checked the photometric consistency between the computed responses in the three ranges B, R1, and R2. For this, we calibrated the three corresponding spectra of one of the standard source, BD+28 4211, observed in the same time interval, and searched for possible discontinuities from range to range. We found no such discontinuity, meaning that the three computed responses are mutually consistent from the point of view of absolute photometry.

The last step in the calibration of the optical spectra was sky background removal. At each wavelength, this background was taken as a linear ramp fitted on two zones of the slit situated on both sides of the nebula and free of nebular or stellar emission. Figure 2 shows the optical spectrum along the whole wavelength range covered, integrated over the 81-pixel (45") zone of the slit where the extinction was computed.

\subsubsection{Estimation of the reddening and of the dereddened $\mathrm{H} \beta$ flux}

Once the spectra were reduced, we first performed an estimation of the profiles of the color excess $E_{B-V}(x)$ and of the dereddened $\mathrm{H} \beta$ flux $F_{\beta}^{\mathrm{der}}(x)$ along the slit, using the case-B line ratios of the hydrogen Balmer decrement and assuming a gas temperature of $11000 \mathrm{~K}$ (Vílchez et al. 1988). We compared the fluxes of the Balmer lines $\mathrm{H} \alpha, \mathrm{H} \beta, \mathrm{H} \gamma$, and $\mathrm{H} \delta$ at the different positions $x$ along the slit common to both ranges $\mathrm{B}$ and $\mathrm{R} 1$. The idea underlying the use of these four lines instead of two is the possibility of correcting the evaluations of $E_{B-V}(x)$ and $F_{\beta}^{\mathrm{der}}(x)$ for the errors in the line fluxes. Such errors were split in three components: photometric residuals, underlying stellar absorption lines, and data noise.

We first measured the fluxes $F_{X}(x)(X=\alpha, \beta, \gamma, \delta)$. For each line and at each position, the flux was measured as the sum of the pixels covered by the line, from which the continuum was previously removed. The fluxes were measured on spectral windows wide enough to include the underlying stellar absorption lines entirely. Then, we applied slight shifts to the line flux profiles, in order to account for object distortions in the frames (see Sect. 2.1.1). We also convolved the $\mathrm{H} \alpha$ profile by a mask so that its PSF along the slit matched the one of the three other lines. Finally, we dereddened the fluxes from foreground Galactic reddening, using the Galactic extinction law (Nandy et al. 1975; Seaton 1979) and $E_{B-V}=0.045$ (Burstein \& Heiles 1984).

Using the LMC law (Howarth 1983) for the extinction due to M33, we then proceeded to correction of the line fluxes for photometric residuals and for underlying stellar absorption lines, using the procedure explained in Appendix A. Finally, from the corrected profiles $F_{X}^{\text {corr }}(x)(X=\alpha, \beta, \gamma)$, we proceeded to the fit of $E_{B-V}(x)$ and $F_{\beta}^{\text {der }}(x)$. The $E_{B-V}(x)$ curve was smoothed with a Gaussian pattern of 6 pixels $F W H M$, because of significant noise in some zones of the slit. On the edges of the nebula, the smoothed curve was slightly negative so we clipped it to 0 . Both $E_{B-V}(x)$ and $F_{\beta}^{\text {der }}(x)$ curves are shown in Fig. 3. As an indication of the quality of the dereddening procedure, Fig. 4 shows the relative residuals $F_{X}^{\text {corr }}(x) / F_{X}^{\text {pred }}(x)-1$, where $F_{X}^{\text {pred }}(x)$ is the flux of $\mathrm{H} X$ predicted from $F_{\beta}^{\operatorname{der}}(x), E_{B-V}(x)$ and the theoretical $\mathrm{H} X / \mathrm{H} \beta$ ratio, for the $\mathrm{H} \alpha, \mathrm{H} \beta$, and $\mathrm{H} \gamma$ lines. Unexplained systematic residuals of a few $\%$ are found for $\mathrm{H} \beta$ and $\mathrm{H} \gamma$ around $x=250$; but by changing artificially the line fluxes, we found that in this part of the 


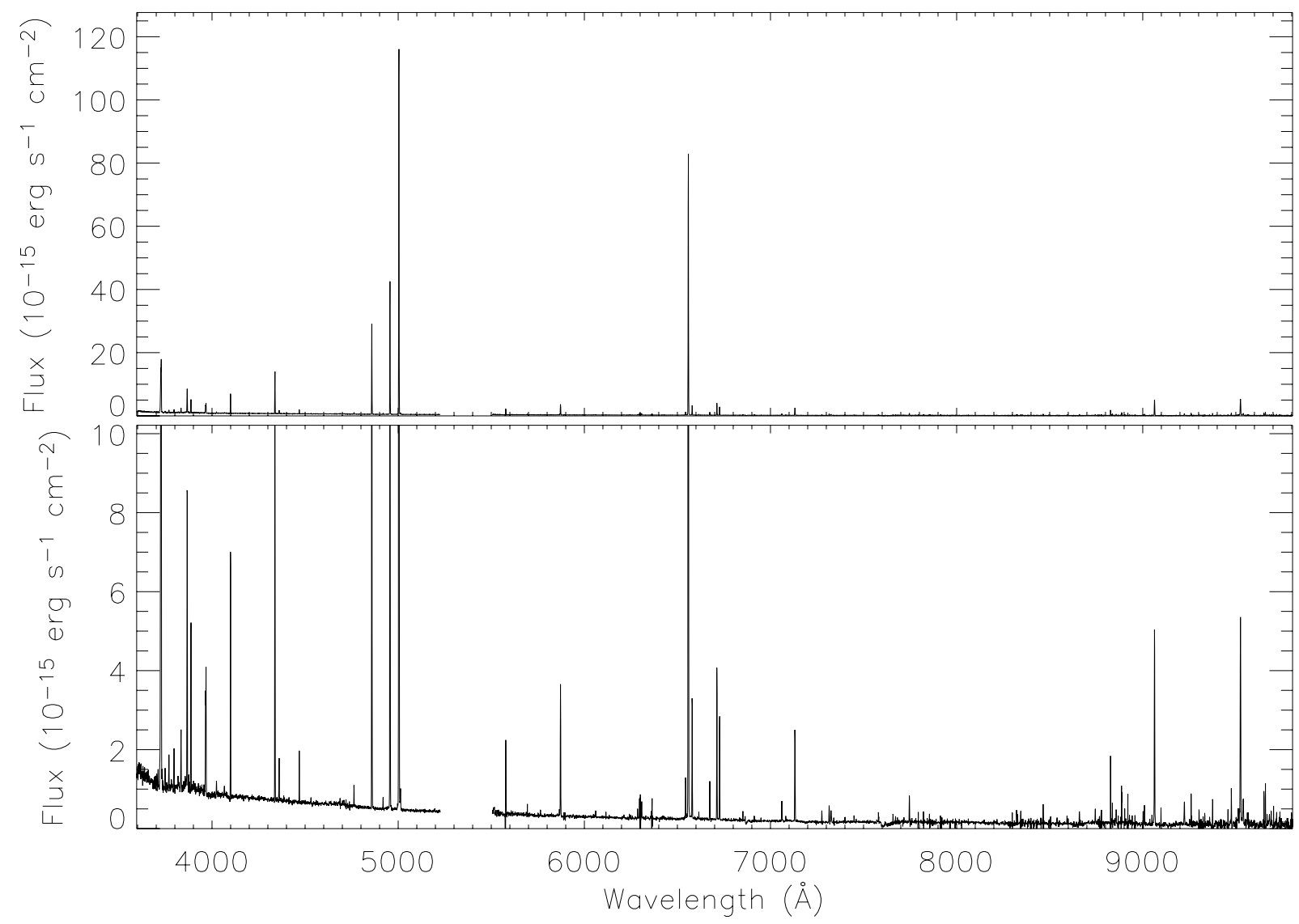

Fig. 2. Optical spectrum of NGC 588 shown in two flux scales.

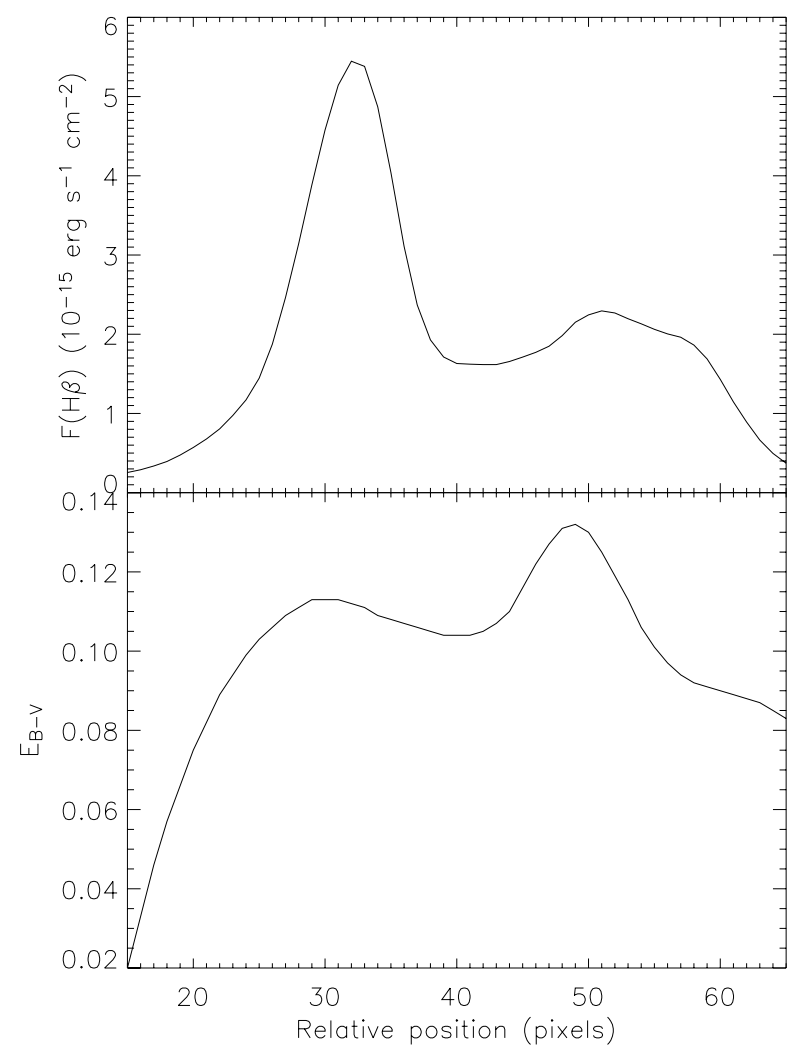

Fig. 3. Curves of $E_{B-V}(x)$ and $F_{\beta}^{\mathrm{der}}(x)$ along the CAHA slit. The $E_{B-V}(x)$ curve does not include the foreground Galactic contribution.

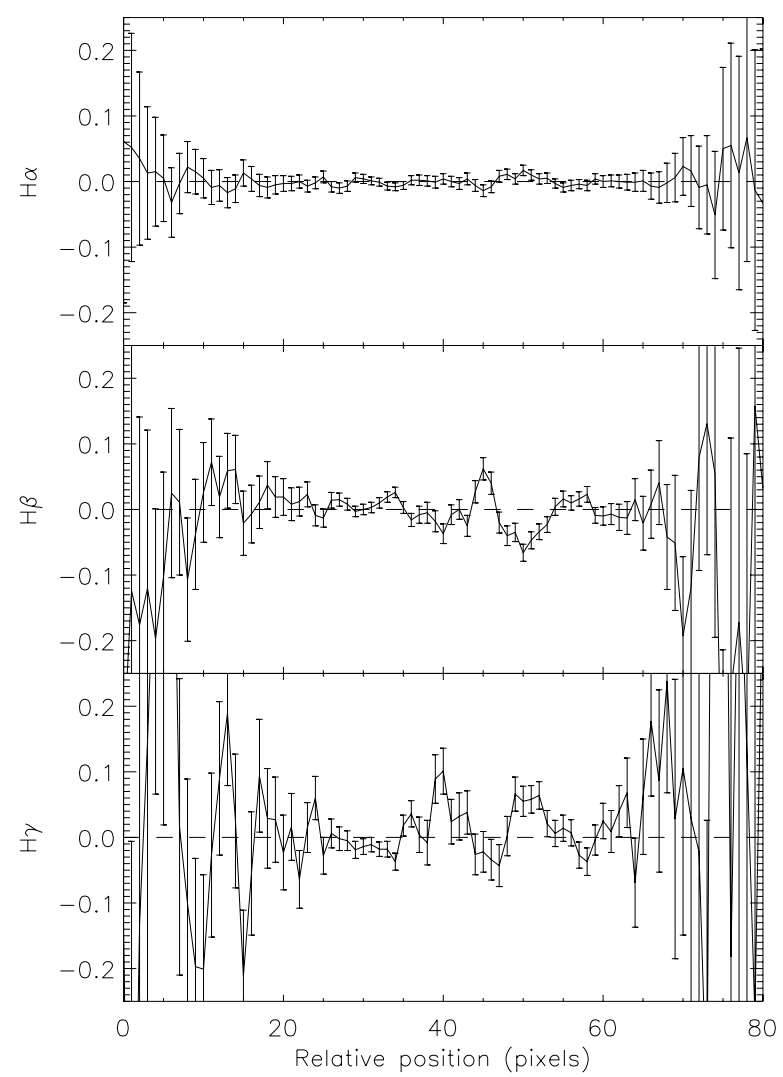

Fig. 4. Residuals of the computation of $F_{\beta}^{\mathrm{der}}(x)$ and $E_{B-V}(x)$. 
slit, the uncertainty on $F_{\beta}^{\text {der }}(x)$ and $E_{B-V}(x)$ is increased by less than $2 \%$ and 0.01 , respectively.

\subsubsection{Line listing and measurement}

We established a list of detected lines from visual inspection of the calibrated frames and selected the ones to be measured. Each line was fitted by a Gaussian curve in a series of bins along the slit. The weakest lines were fitted in bins of several pixels whose sizes were ruled by their overall signalto-noise ratios $(\mathrm{S} / \mathrm{N})$, so as to ensure the convergence of the Gaussian curve fits. The fits were performed with the program TWODSPEC of the STARLINK ${ }^{2}$ software suite; the [OII] $\lambda 3726+3729$ doublet and of the [OI] $\lambda 6300$ line, blended with an emission line of the sky, were measured with a personal IDL routine. Each bin was then dereddened using the corresponding value of $E_{B-V}$. Finally, to obtain the line intensities with respect to $\mathrm{H} \beta$, we summed the dereddened line fluxes in all the bins and divided the result by the dereddened $\mathrm{H} \beta$ flux summed over the same bins. In this procedure, we included the bins where $E_{B-V}$ was clipped to 0 .

\subsubsection{Detection limit and accuracy of the measured fluxes}

From measurements of the noise in the frames, we estimated the $3 \sigma$ detection limit of emission lines in the spectra. We found this limit to vary approximately from $F(\mathrm{H} \beta) / 2000(\lambda \geq$ $5000 \AA)$ to $F(\mathrm{H} \beta) / 500(\lambda \approx 3700 \AA)$. The $\mathrm{S} / \mathrm{N}$ was then very high for the main lines. However, recombination lines of metals remained undetectable. In particular, the $3 \sigma$ detection threshold for the CII $\lambda 4267$ and OII $\lambda 4651$ was $\approx F(\mathrm{H} \beta) / 500$.

As already mentioned, in each spectral range, we found oscillations of $\sim 3 \%$ amplitude in the residuals of the fit of the photometric instrumental response. These oscillations are even higher below $\lambda=4000 \AA$, causing a $\approx 15 \%$ uncertainty in the photometric match of this range with the rest of the $\mathrm{B}$ range. Hence, when calculating line intensity ratios, we decided to add $3 \%$ of uncertainty in the fluxes of the lines with $\lambda>4000 \AA$, and $15 \%$ for lines with $\lambda \leq 4000 \AA$. There are two exceptions to this rule: the $\mathrm{H} \beta$ line (see Sect. 2.1.2) and the ratios of lines belonging to a close doublet and then suffering identical photometric errors (e.g., [OII] $\lambda 3726 / \lambda 3729$, [SII] $\lambda 6731 / \lambda 6716$ ).

Telluric absorption bands are another important source of uncertainty. The way we corrected the calibration spectra for their effect is very approximate and let in an uncertainty of $\sim 30 \%$ at $\lambda>8200 \AA$ A. However, the main source of error in this range comes from the lack of knowledge about the coincidence of the individual (unresolved) lines of the bands with the emission lines of the nebula, especially [SIII] $\lambda 9069$ and [SIII] 29532. Indeed, the flux of a nebular line can be severely reduced, depending on its exact position with respect to the telluric absorption lines. Consequently, we did not use the fluxes of the lines situated at $\lambda>8200 \AA$.

${ }^{2}$ The STARLINK Project is run by CCLRC on behalf of PPARC. TWODSPEC was developed by T. N. Wilkins and D. J. Axon.
Table 2. Dereddened fluxes of the lines and doublets used in this work, in units of $\mathrm{H} \beta=100$, and associated measure standard deviations in the same units. ${ }^{a}$ Fluxes corrected for the SWS apertures.

\begin{tabular}{lll}
\hline \hline Line/doublet & Flux & Standard deviation \\
\hline Optical slit & & \\
\hline [OII] $\lambda 372(6+9)$ & 141.54 & 0.36 \\
[NeIII] $\lambda 3869$ & 36.86 & 0.18 \\
[SIII $\lambda 40(69+76)$ & 1.58 & 0.14 \\
[OIII $\lambda 4363$ & 3.67 & 0.07 \\
[ArIV] $\lambda 4711$ & 0.26 & 0.07 \\
[ArIV] $\lambda 4741$ & 0.34 & 0.05 \\
[OIII] $\lambda 5007$ & 404.79 & 0.20 \\
[NII] $\lambda 5755$ & 0.20 & 0.03 \\
HeI $\lambda 5876$ & 11.94 & 0.04 \\
[SIII] $\lambda 6312$ & 1.38 & 0.04 \\
[NII] $\lambda 6583$ & 10.69 & 0.04 \\
[SII] $\lambda 6716$ & 12.73 & 0.04 \\
[SII] $\lambda 6731$ & 9.17 & 0.03 \\
[ArIII $\lambda 7135$ & 9.47 & 0.03 \\
[OII] $\lambda 73(20+30)$ & 3.09 & 0.05 \\
\hline Integrated fluxes & & \\
\hline [OIII] $\lambda 5007$ & 352.22 & 12.46 \\
[SIV] $\lambda 11 \mu \mathrm{m}^{a}$ & 37.81 & 9.72 \\
[NeIII] $\lambda 16 \mu \mathrm{m}^{a}$ & 56.51 & 4.75 \\
[SIII] $\lambda 19 \mu \mathrm{m}^{a}$ & 81.42 & 13.66 \\
[SIII] $\lambda 33 \mu \mathrm{m}^{a}$ & 56.23 & 9.73 \\
[OIII] $\lambda 88 \mu \mathrm{m}$ & 214.47 & 8.18 \\
\hline
\end{tabular}

Finally, an additional uncertainty in each line ratio results from a constant uncertainty of 0.02 in the $E_{B-V}$ curve. Table 2 presents the reddening-corrected intensities of all the lines measured in the spectrum and used in this work, in units of $\mathrm{H} \beta=100$, and the associated standard deviations (i.e. the uncertainties related to random errors only).

\subsection{Ground-based images}

We retrieved several narrow-band images of the ING archive. They were obtained through filters centered on $\mathrm{H} \alpha, \mathrm{H} \beta$, [OIII] $\lambda 5007$ and on the red side of the $\mathrm{H} \alpha$ continuum, at the wavelength $\lambda=6384 \AA$. The $\mathrm{H} \alpha$ filter is also transparent to the [NII] $\lambda 65(48+83)$ doublet, but the latter contributes little to the signal of the images and was neglected.

Using some stars common to all the images, we subtracted the $\mathrm{H} \alpha$ continuum from the three other images, in order to keep only the signal of the nebular emission lines. We then established a reddening map, as shown in Fig. 5. This map was obtained after smoothing the $\mathrm{H} \alpha$ and $\mathrm{H} \beta$ images with a $31 \times 31$ pixel mask, due to the small S/N ratio of the latter image. It can be seen that the reddening in the nebula is quite uniform, so we replaced it with a constant reddening. This simplification causes an error of only $\sim 1 \%$ in the computation of the total dereddened $\mathrm{H} \beta$ flux. We also established an [OIII] $\lambda 5007 / \mathrm{H} \alpha$ map, as shown in Fig. B.2 and discussed in Appendix B.

Knowing the position of the optical slit on the nebula and the $\mathrm{H} \beta$ flux measured through it, and assuming a distance of $800 \mathrm{kpc}$ to the nebula (Lee et al. 2002), we calculated the 

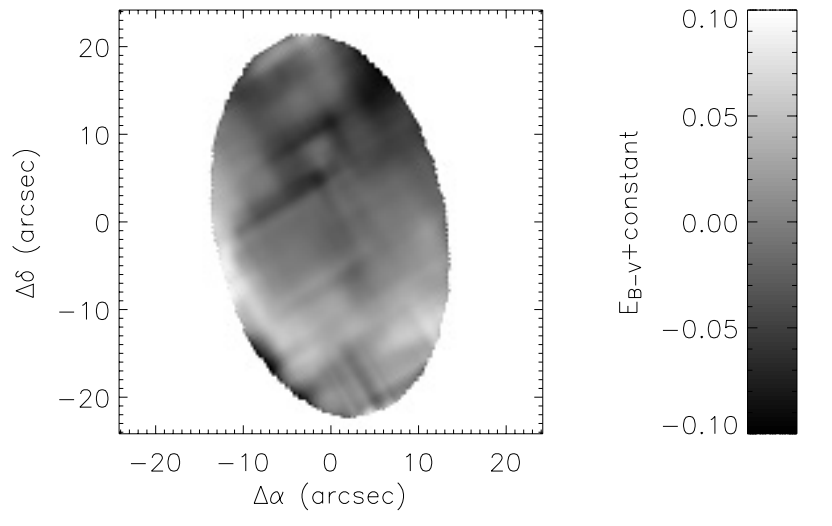

Fig. 5. Map of $E_{B-V}$ established with the continuum-free $\mathrm{H} \alpha$ and $\mathrm{H} \beta$ images. The average uncertainty of this map is $\approx 0.03$. The computed reddening outside the ellipse cutout is less accurate, so it is not shown here.

total extinction-corrected $\mathrm{H} \beta$ luminosity of NGC 588: $L_{\beta}=$ $1.22 \times 10^{38} \mathrm{erg} \mathrm{s}^{-1}$. We tested this luminosity by using the radio flux density at $1.4 \mathrm{GHz}$ measured by Viallefond et al. (1986). The observed radio-to- $\mathrm{H} \beta$ ratio is $F_{\nu}(1.4 \mathrm{GHz}) / F_{\beta}=$ $(3.3 \pm 0.2) \times 10^{-14} \mathrm{~Hz}^{-1}$. We used the emissivity formulae of Péquignot et al. (1991) for $\mathrm{H} \beta$ and of Osterbrock (1974) for the radio continuum. In the latter, we included the free-free emissions induced by the $\mathrm{H}^{+}$and $\mathrm{He}^{+}$ions. Adopting a temperature of $11000 \mathrm{~K}$ and an $\mathrm{He}^{+} / \mathrm{H}^{+}$abundance ratio of 0.083 (Vílchez et al. 1988), we found a theoretical ratio $F_{v}(1.4 \mathrm{GHz}) / F_{\beta}=$ $3.66 \times 10^{-14} \mathrm{~Hz}^{-1}$, a value higher by $10 \%$ than the observed one. The theoretical value of $F_{\nu}(1.4 \mathrm{GHz}) / F_{\beta}$ depends little on the temperature, and we can assess that the total dereddened $\mathrm{H} \beta$ flux that we found is consistent with the measurements of Viallefond et al. (1986) to within $10 \%$. Consequently, for comparison with the ISO data, we used our value of $L_{\beta}$ with an associated uncertainty of $10 \%$.

\subsection{ISO line spectra}

A number of infrared (IR) lines were observed with the Infrared Space Observatory (ISO), in the ranges of both the Largeand Small-Wavelength Spectrometers (LWS and SWS, respectively). We retrieved 3 data sets from LWS observations and 1 from SWS observations (Table 1).

The LWS data sets 80800268 and 81601776 were analyzed by Higdon et al. (2003), who found a value of $3.51 \times$ $10^{-12} \mathrm{erg} \mathrm{s}^{-1} \mathrm{~cm}^{-2}$ for the [OIII] $\lambda 88 \mu \mathrm{m}$ flux. Given the important constraint provided by this line in our study, we performed our own measurements on the three available data sets. For this, we used the ISAP $^{3}$ facility. For each spectrometer and each detector (\#4 and \#5 for LWS01, \#5 for LWS02), we obtained one spectrum by selecting the scans to combine and by removing bad pixels. Even when processed, the LWS02 contained spurious features, so it was rejected. The measurements of the [OIII] $\lambda 88 \mu \mathrm{m}$ flux on the four LWS01

\footnotetext{
${ }^{3}$ The ISO Spectral Analysis Package (ISAP) is a joint development by the LWS and SWS Instrument Teams and Data Centers. Contributing institutes are CESR, IAS, IPAC, MPE, RAL and SRON.
}
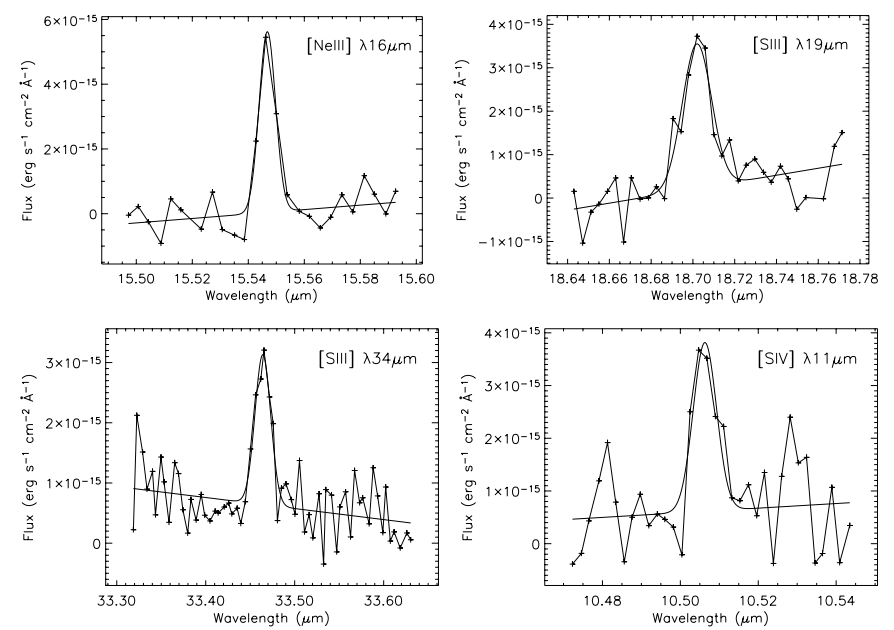

Fig. 6. ISO-SWS detected lines.

spectra gave compatible values. Combining them, we find a flux of $(3.41 \pm 0.13) \times 10^{-12} \mathrm{erg} \mathrm{s}^{-1} \mathrm{~cm}^{-2}$, in agreement with the value given by Higdon et al. (2003). In the following we adopted our value. Accounting for the estimates given in the LWS Handbook (Gry et al. 2003) on the repeatability of the LWS01 observations and the absolute photometric accuracy of this spectrometer, we estimate that the overall uncertainty in the [OIII] $\lambda 88 \mu \mathrm{m}$ flux is $20 \%$. Since the LWS aperture ( $\left.84^{\prime \prime}\right)$ is much larger than the nebula, no aperture correction was needed for the [OIII] $\lambda 88 \mu \mathrm{m}$ flux.

The SWS spectra were processed with the ISAP facility in the same way as the LWS data. We measured the fluxes of the lines [NeIII] $\lambda 16 \mu \mathrm{m}$, [SIII] $\lambda 19 \mu \mathrm{m}$, [SIII] $\lambda 33 \mu \mathrm{m}$, and [SIV] $\lambda 11 \mu \mathrm{m}$ (Fig. 6). The SWS apertures range from $14^{\prime \prime} \times 20^{\prime \prime}$ to $33^{\prime \prime} \times 20^{\prime \prime}$ and are therefore smaller than the nebula. To estimate the total nebular fluxes in each of these lines, we assumed that their surface brightness distribution is identical to the one observed in the $\mathrm{H} \alpha$ image. This assumption is not entirely correct because of ionization stratification of the nebula. We tested it with a few photoionization models, using various ionizing spectra and density distributions, and found that it leads to an error of $\approx 10 \%$ at most. Considering Table 5.3 of the SWS Handbook (Leech et al. 2003), we adopted an instrumental uncertainty of $20 \%$ in addition to the dispersions in the line fits and the error in the aperture corrections.

The aperture-corrected intensities of the ISO detected lines are reported in Table 2, together with their non-instrumental uncertainties and the aperture correction factors. They are given relative to the total $\mathrm{H} \beta$ flux of the nebula (Sect. 2.2).

\section{Empirical diagnostics}

\subsection{Density and temperature}

A series of temperature and density diagnostics are available from our observed line flux ratios. We established a $\left(T_{\mathrm{e}}, \log N_{\mathrm{e}}\right)$ curve for five temperature and three density diagnostics, as seen in Fig. 7. In this diagram, a $7 \%$ uncertainty was set to the [SII] $\lambda 6731 / \lambda 6716$ ratio, in order to account for the 


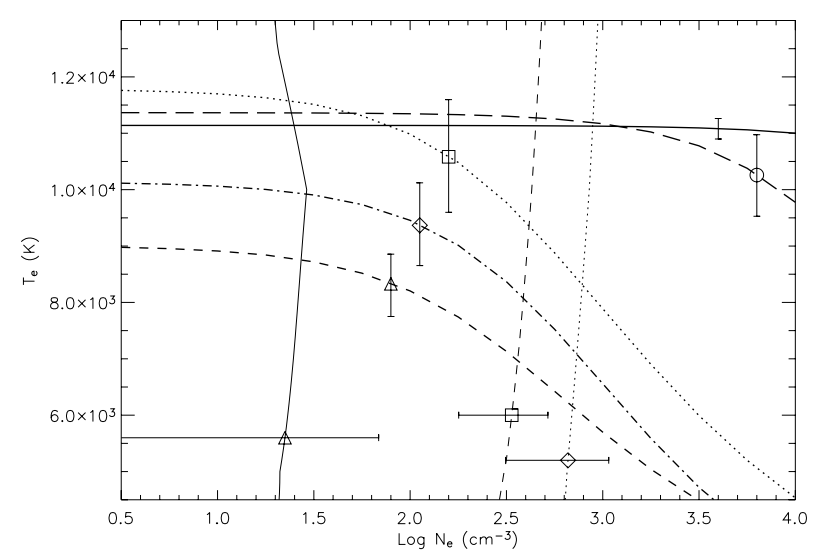

Fig. 7. $\left(T_{\mathrm{e}}, \log N_{\mathrm{e}}\right)$ diagram for the following ratios. Temperature diagnostics (with vertical error bars): [OIII] $\lambda 4363 / \lambda 5007$ (full line, no symbol), [OII] $\lambda 73(20+30) / \lambda 372(6+9)($ dotted line, square), [SII] $\lambda 40(69+76) / \lambda 67(16+31)$ (dot-dashed line, diamond), [NII] $\lambda 5755 / \lambda 6583$ (long-dashed line, circle), [OIII] $\lambda 5007 / \lambda 88 \mu \mathrm{m}$ (shortdashed line, triangle). Density diagnostics (with horizontal error bars): [SII] $\lambda 6731 / \lambda 6716$ (full line, triangle), [OII] $\lambda 3726 / \lambda 3729$ (shortdashed line, square), [SIII] $\lambda 19 \mu \mathrm{m} / \lambda 34 \mu \mathrm{m}$ (dotted line, diamond). The error bars shown here are representative of the whole $\log N_{\mathrm{e}} / T_{\mathrm{e}}$ ranges.

Table 3. Empirical temperatures derived from five line ratios.

\begin{tabular}{ll}
\hline \hline Line ratio & Temperature $(\mathrm{K})$ \\
\hline$[\mathrm{OIII}] \lambda 4363 / \lambda 5007$ & $11140 \pm 180$ \\
{$[\mathrm{OII}] \lambda \lambda 7320+30 / \lambda 3727$} & $11210 \pm 1180$ \\
{$[\mathrm{SII}] \lambda \lambda 4069+76 / \lambda \lambda 6716+31$} & $9860 \pm 940$ \\
{$[\mathrm{NII}] \lambda 5755 / \lambda 6583$} & $11360 \pm 840$ \\
{$[\mathrm{OIII}] \lambda 5007 / \lambda 88 \mu \mathrm{m}$} & $8420 \pm 690$ \\
\hline
\end{tabular}

uncertainties in the collisional strengths of this ion. Likewise, the [OII] $\lambda 3726 / \lambda 3729$ ratio uncertainty was set to $15 \%$.

At temperatures close to $10000 \mathrm{~K}$, a simple least-square fit of the [OII] $\lambda 3726 / \lambda 3729$, [SIII] $\lambda 19 \mu \mathrm{m} / \lambda 34 \mu \mathrm{m}$ and [SIII] $\lambda 19 \mu \mathrm{m} / \lambda 34 \mu \mathrm{m}$ line ratios with a single electron density yields $N_{\mathrm{e}}=70 \pm 60 \mathrm{~cm}^{-3}$. However, while the [OII] and [SIII] ratios indicate very similar densities, the [SII] ratio indicates a smaller one. This is not necessarily inconsistent, since [SII] lines are emitted further from the ionizing source than [SIII] and [OII] lines, so that the electron density indicated by the [SII] doublet is expected to be smaller in case of an outward-decreasing gas density. Figure 8 shows the [SII] $\lambda 6731 / \lambda 6716$ and [OII] $\lambda 3726 / \lambda 3729$ ratio profiles along the slit with respect to the $\mathrm{H} \beta$ flux distribution. Both ratios are increasing functions of the electron density. While the [OII] profile is nearly constant, the [SII] one suggests a decrease in the outskirts of the nebula. However, in the low-density regime we observe here, interpretation of the [SII] and [OII] profiles is not fully conclusive. The $\mathrm{H} \beta$ flux is another density indicator and we use it in Sect. 6.1.1.

Taking an electron density of $70 \mathrm{~cm}^{-3}$, we derived empirical temperatures from the five corresponding diagnostics. The values are listed in Table 3. As seen, the four diagnostics using only optical lines, $T_{\text {opt }}(\mathrm{OIII}), T(\mathrm{OII}), T(\mathrm{SII})$, and $T(\mathrm{NII})$, are in relatively good agreement with a mean temperature

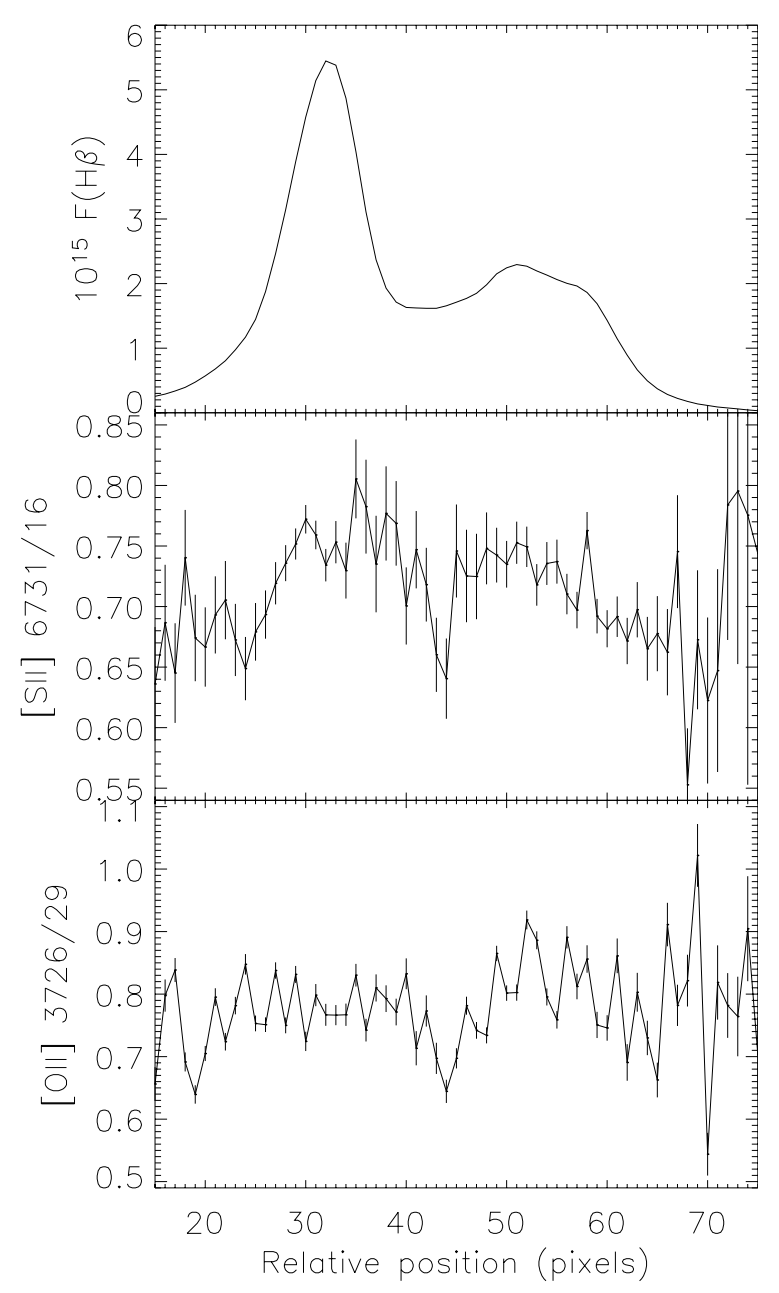

Fig. 8. [SII] $\lambda 6731 / \lambda 6716$ and [OII] $\lambda 3726 / \lambda 3729$ ratio profiles along the slit. The $\mathrm{H} \beta$ flux profile is shown in the upper panel.

of $\approx 11000 \mathrm{~K}, T_{\text {opt }}(\mathrm{OIII})$ being by far the most constraining of the four diagnostics. However, the [OIII] $\lambda 5007 / \lambda 88 \mu \mathrm{m}$ ratio gives a temperature $T_{\mathrm{IR}}(\mathrm{OIII})$ that is lower by $\approx 3000 \mathrm{~K}$ than $T_{\text {opt }}(\mathrm{OIII})$. This discrepancy cannot be attributed to the error bars alone. Indeed, to yield a temperature of $11000 \mathrm{~K}$, the [OIII] $\lambda 5007 / \lambda 88 \mu \mathrm{m}$ ratio would have to be 3.6 instead of 1.6. Such a large discrepancy cannot be attributed to errors of measurement and/or calibration. Instead, it suggests the presence of large temperature inhomogeneities in the nebula.

The discrepancy between the optical temperature diagnostics and $T_{\mathrm{IR}}(\mathrm{OIII})$ might arise from two phenomena: (i) the optical slit samples a peculiar zone of plasma hotter than the overall gas of the nebula, making the temperature diagnostics, representative of this zone higher than $T_{\mathrm{IR}}(\mathrm{OIII})$ which is measured on the whole nebula; or (ii) temperature variations exist throughout the entire nebula. In the latter case, for each of the optical temperature diagnostics, the more temperaturesensitive line (e.g., [OIII] 24363) significantly weights the hotter plasma zones, biasing the temperature measure toward a higher than average value. This bias also affects $T_{\mathrm{IR}}(\mathrm{OIII})$ but to a lower degree, hence making it smaller than the temperatures derived from optical line ratios. 
Table 4. Empirical abundance ratios of various elements, with the ICFs specified.

\begin{tabular}{lll}
\hline \hline \multicolumn{2}{l}{ Ratio } & \multicolumn{2}{l}{ Value } & ICF \\
\hline \multicolumn{2}{l}{ Our work } & \\
\hline $\mathrm{He} / \mathrm{H}$ & $(8.96 \pm 0.42) \times 10^{-2}$ & $\mathrm{He}=\mathrm{He}^{+}+\mathrm{He}^{++}$ \\
$\mathrm{O} / \mathrm{H}$ & $(1.45 \pm 0.27) \times 10^{-4}$ & $\mathrm{O}=\mathrm{O}^{+}+\mathrm{O}^{++}$ \\
$\mathrm{N} / \mathrm{O}$ & $(4.12 \pm 0.95) \times 10^{-2}$ & $\mathrm{~N} / \mathrm{O}=\mathrm{N}^{+} / \mathrm{O}^{+}$ \\
$\mathrm{Ne} / \mathrm{O}$ & $(2.57 \pm 0.14) \times 10^{-1}$ & $\mathrm{Ne} / \mathrm{O}=\mathrm{Ne}^{++} / \mathrm{O}^{++}$ \\
\hline Vílchez et al. $(1988)$ & \\
\hline $\mathrm{He} / \mathrm{H}$ & $8.3 \times 10^{-2}$ & \\
$\mathrm{O} / \mathrm{H}$ & $(2.0 \pm 0.3) \times 10^{-4}$ & \\
$\mathrm{~N} / \mathrm{O}$ & $(3.0 \pm 0.5) \times 10^{-2}$ & \\
$\mathrm{Ne} / \mathrm{O}$ & $(2.5 \pm 0.5) \times 10^{-1}$ & \\
\hline
\end{tabular}

Assuming that the discrepancy between $T_{\text {opt }}(\mathrm{OIII})$ and $T_{\text {IR }}(\mathrm{OIII})$ is not a sampling effect, we can estimate the average $T_{0}$ and the mean square fluctuations $T_{0}^{2} t^{2}$ of the temperature in the $\mathrm{O}^{++}$zone, both weighted by $N_{\mathrm{e}} N\left(\mathrm{O}^{++}\right)$. By expanding to order 2 the sensivity of the lines to the temperature and assuming a density of $70 \mathrm{~cm}^{-3}$, we found $T_{0}=8110 \pm 660 \mathrm{~K}$ and $t^{2}=0.087 \pm 0.008$, i.e. the temperature undergoes fluctuations of about $30 \% \mathrm{rms}$. This amplitude of fluctuations is larger than those generally found in HII regions (typical values of $t^{2}$ are 0.02-0.05: Esteban et al. 2002).

\subsection{Abundances}

We computed a few empirical elemental abundance ratios, using $N_{\mathrm{e}}=70 \mathrm{~cm}^{-3}$ and the $\mathrm{O}^{+}$and $\mathrm{O}^{++}$optical temperature diagnostics. The ionic abundances were derived from lines measured in the optical spectra. The values and the ionization correction factors (ICFs) used are summarized in Table 4. The error bars account for the uncertainties in the adopted density, in the empirical temperatures and in the line fluxes. The abundances are comparable to the ones inferred by Vílchez et al. (1988). We found the $\mathrm{O} / \mathrm{H}$ value to be $30 \%$ of the solar one as given by Lodders (2003). At this stage, we did not compute the abundances of elements such as S or Ar, due to the uncertainties in their ICFs.

The empirical elemental abundance ratios presented here served as a starting point for our modeling of the nebula.

\section{General strategy for model fitting}

To model the nebula, we used the code PHOTO (Stasińska 1990) with the update of atomic data listed in Stasińska (2005). The abundance ratios $\mathrm{Mg} / \mathrm{O}, \mathrm{Si} / \mathrm{O}, \mathrm{S} / \mathrm{O}, \mathrm{Cl} / \mathrm{O}, \mathrm{Ar} / \mathrm{O}, \mathrm{Fe} / \mathrm{O}$ and $\mathrm{C} / \mathrm{N}$ were set to the solar values quoted from Lodders (2003). The input ionizing spectra were those inferred by Jamet et al. (2004) in their star-by-star analysis of the cluster, for an age ranging from 3.6 to $4.4 \mathrm{Myr}$.

The optical slit that we used is thin compared to the nebula, and we can consider that the line fluxes measured in the optical spectra are representative of a plane section in the nebula, perpendicular to the sky. Consequently, those lines were compared to model line intensities integrated in this plane slice. In the case of the line fluxes representative of the whole nebula ([OIII] $\lambda 5007 / \mathrm{H} \beta$ ratio from the images, lines observed with ISO), the model line intensities were integrated into the whole gas volume.

Table 5 summarizes the line ratios used to constrain the models, sorted according to the data they mainly diagnose. The order of the lines in each ratio was chosen so that the ratio is an increasing function of the quantity it probes. We considered that satisfactory models should be able to reproduce all those constraints simultaneously. The observed values are quoted with their relative tolerances in percentile of the observed values. For most of the line ratios, those tolerances were defined as the $1 \sigma$ measure uncertainties. The exceptions are the following. We set the tolerances on [SII] $\lambda 6731 / \lambda 6716$ and [OII] $\lambda 3726 / \lambda 3729$ to $7 \%$ and $15 \%$, respectively, due to the uncertainties in the collisional strengths of the regarded transitions. We also increased by $25 \%$ the tolerance for the [SIII] $\lambda 6312 /[\mathrm{SII}] \lambda 67(17+31)$ and [SIV] $\lambda 11 \mu \mathrm{m} /[\mathrm{SIII}] \lambda(19+34) \mu \mathrm{m}$ diagnostics, in order to account for the poorly known dielectronic coefficients of sulfur. In Table 5, the line ratios used as temperature diagnostics are followed by the corresponding empirical temperatures for a density $N_{\mathrm{e}}=70 \mathrm{~cm}^{-3}$.

\section{Simple models}

\subsection{Full sphere}

We first modeled the nebula in a very simple way. We assumed it to be a full sphere of constant density with a radius $R=1.5 \times$ $10^{20} \mathrm{~cm}$ which approximately fits the orientation-averaged size of the bright annulus that can be seen in the $\mathrm{H} \alpha$ image (Fig. 1). The input ionizing spectrum was the one obtained for an age of 4.2 Myr, the best-fit age from Jamet et al. (2004). We used the total $\mathrm{H} \beta$ luminosity of the nebula to constrain the relation between the filling factor $\epsilon$ and the hydrogen density $N_{\mathrm{H}}$.

We considered several values of $\epsilon$. For $\epsilon=1$, which leads to the smallest possible density, the model (labelled [FS] in Table 5) produced [OIII]/[OII], [SIII]/[SII] and [SIV]/[SIII] ratios that agree satisfactorily with the data but have too a small value of [ArIV]/[ArIII]. For models with $\epsilon<1$, all four excitation diagnostic line ratios were underestimated. However, the main failure of the full sphere model is that it does not conciliate the temperature diagnostics based on optical lines and the [OIII] optical/IR one. Indeed, it predicts a total amplitude of the variations of the electronic temperature $T_{\mathrm{e}}$ of $\approx 1000 \mathrm{~K}$ only.

\subsection{Hollow bubble}

The $\mathrm{H} \alpha$ image (Fig. 1) shows that the nebula is better represented by a bubble than by a full sphere. Hence, we modeled the nebula as a hollow bubble whose thickness is $20 \%$ of its average radius $R=1.5 \times 10^{20} \mathrm{~cm}$. We assumed $\epsilon=1$, leading to a density $N_{\mathrm{H}}=11 \mathrm{~cm}^{-3}$ (derived from the $\mathrm{H} \beta$ luminosity). As seen in Table 5, this model, labelled [HB], fails to reproduce $T_{\text {opt }}(\mathrm{OIII})$, while it marginally agrees with $T_{\mathrm{IR}}(\mathrm{OIII})$. It also underestimates the excitation of the nebula. 
Table 5. Observational values of the line ratios used to constrain the photoionization models and model results in the form (model-observation)/(tolerance). The models are: [FS] Full Sphere; [HB] Hollow Bubble; [DD1] and [DD2] H $\beta$ constrained Density Distribution, 4.2 Myr spectrum; [DD2] H $\beta$ constrained Density Distribution, 3.6 Myr Spectrum; [DF] Density Fluctuations; [DG] Dust Grains. The geometric corrections for the models of Sect. 6 and further sections are also written. ${ }^{a}$ Values are valid for the whole nebula; the other data are representative of the optical slit.

\begin{tabular}{|c|c|c|c|c|c|c|c|c|}
\hline Diagnostics & Observed & [FS] & 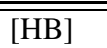 & $\overline{~[\mathrm{DD} 1]}$ & [DD2] & [DDS] & 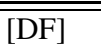 & 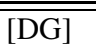 \\
\hline Section & & 5.1 & 5.2 & 6.2 & 6.2 & 7.1 & 7.2 & 7.3 \\
\hline \multicolumn{9}{|l|}{ Density } \\
\hline$[\mathrm{SII}] \lambda 6731 / \lambda 6716$ & $0.72 \pm 7 \%$ & -0.1 & +0.0 & -0.1 & +0.0 & +0.2 & +0.1 & +0.7 \\
\hline [OII] $\lambda 3726 / \lambda 3729$ & $0.78 \pm 15 \%$ & -2.1 & -2.1 & -2.1 & -2.1 & -2.0 & -2.1 & -1.9 \\
\hline$[\mathrm{SIII}] \lambda 19 \mu \mathrm{m} / \lambda 34 \mu \mathrm{m}^{a}$ & $1.45 \pm 37 \%$ & -1.8 & -1.8 & -1.8 & -1.8 & -1.8 & -1.8 & -1.8 \\
\hline \multicolumn{9}{|l|}{ Temperature } \\
\hline [OIII] $\lambda 4363 / \lambda 5007$ & $0.0091 \pm 5 \%$ & -5.5 & -4.9 & -5.4 & -1.6 & +0.2 & -1.6 & +4.5 \\
\hline [OII] $\lambda 73(20+30) / \lambda 372(6+9)$ & $0.0218 \pm 17 \%$ & -1.0 & -1.1 & -1.0 & -0.4 & -0.2 & -0.4 & -0.2 \\
\hline$[\mathrm{SII}] \lambda 40(69+76) / \lambda 67(16+31)$ & $0.0722 \pm 11 \%$ & -2.3 & -2.3 & -2.3 & -1.7 & -1.6 & -1.8 & -1.9 \\
\hline$[\mathrm{NII}] \lambda 5755 / \lambda 6583$ & $0.0187 \pm 16 \%$ & -1.2 & -1.3 & -1.2 & -0.3 & -0.2 & -0.5 & -0.6 \\
\hline [OIII] $\lambda 5007 / \lambda 88 \mu \mathrm{m}^{a}$ & $1.64 \pm 23 \%$ & +2.1 & +2.3 & +2.4 & +3.9 & +4.4 & +3.3 & +5.6 \\
\hline$T_{\text {opt }}(\mathrm{OIII})(\mathrm{K})$ & $11140 \pm 180$ & 10080 & 10210 & 10100 & 10840 & 11850 & 10850 & 11950 \\
\hline$T(\mathrm{OII})(\mathrm{K})$ & $11210 \pm 1180$ & 10130 & 9990 & 10160 & 10780 & 11030 & 10740 & 10950 \\
\hline$T(\mathrm{SII})(\mathrm{K})$ & $9860 \pm 940$ & 8060 & 8050 & 8070 & 8490 & 8630 & 8440 & 8390 \\
\hline$T(\mathrm{NII})(\mathrm{K})$ & $11360 \pm 840$ & 10380 & 10300 & 10380 & 11080 & 11230 & 10960 & 10840 \\
\hline$T_{\mathrm{IR}}(\mathrm{OIII})(\mathrm{K})^{a}$ & $8420 \pm 690$ & 9470 & 9600 & 9640 & 10320 & 10550 & 10080 & 11090 \\
\hline \multicolumn{9}{|l|}{ Excitation } \\
\hline [OIII] $\lambda 5007 /[\mathrm{OII}] \lambda(3726+9)$ & $2.86 \pm 16 \%$ & -1.7 & -4.9 & -0.4 & +0.2 & -0.2 & -0.3 & -0.3 \\
\hline$[\mathrm{SIII}] \lambda 6312 /[\mathrm{SII}] \lambda(6716+31)$ & $0.0631 \pm 25 \%$ & +2.0 & -1.1 & +6.3 & +9.7 & +4.9 & +3.2 & +1.5 \\
\hline$[$ ArIV] $\lambda(4711+41) /[$ ArIII $] \lambda 7135$ & $0.074 \pm 15 \%$ & -3.9 & -6.4 & -3.9 & -3.4 & -1.2 & -0.3 & +2.9 \\
\hline$[\mathrm{SIV}] \lambda 11 \mu \mathrm{m} /[\mathrm{SIII}] \lambda(19+34) \mu \mathrm{m}^{a}$ & $0.270 \pm 37 \%$ & -1.0 & -2.4 & -0.9 & -0.9 & -1.3 & -0.8 & +0.1 \\
\hline \multicolumn{9}{|l|}{ Abundances } \\
\hline HeI $\lambda 5876 / \mathrm{H} \beta$ & $0.1193 \pm 5 \%$ & -0.7 & -1.2 & -0.1 & +0.0 & -0.1 & +0.0 & -0.3 \\
\hline [OIII] $\lambda 5007 / \mathrm{H} \beta$ & $4.05 \pm 4 \%$ & -7.1 & -13.3 & -5.9 & -0.8 & +0.9 & -1.5 & +2.2 \\
\hline [NII] $\lambda 6563 /[\mathrm{OII}] \lambda(3726+9)$ & $0.075 \pm 16 \%$ & -0.1 & +0.0 & -0.4 & -1.1 & -1.0 & -0.6 & -0.3 \\
\hline [NeIII] $\lambda 3869 /[\mathrm{OIII}] \lambda 5007$ & $0.0911 \pm 5 \%$ & -4.3 & -6.0 & -4.7 & -4.0 & -0.5 & -0.2 & +1.0 \\
\hline$[$ ArIII $] \lambda 7135 / \mathrm{H} \beta$ & $0.0946 \pm 5 \%$ & +1.7 & +1.7 & +2.7 & +6.9 & +7.9 & +5.6 & +7.2 \\
\hline [OIII] $\lambda 88 \mu \mathrm{m} / \mathrm{H} \beta^{a}$ & $2.21 \pm 25 \%$ & -2.2 & -2.8 & -2.1 & -2.0 & -2.0 & -1.9 & -2.1 \\
\hline$[\mathrm{NeIII}] \lambda 16 \mu \mathrm{m} /[\mathrm{OIII}] \lambda 88 \mu \mathrm{m}^{a}$ & $0.257 \pm 33 \%$ & +0.2 & -0.1 & +0.3 & +0.3 & +1.0 & +0.9 & +1.5 \\
\hline \multicolumn{9}{|l|}{ Other } \\
\hline [OI] $\lambda 6300 / \mathrm{H} \beta$ & $0.02 \pm 50 \%$ & -1.6 & -1.0 & -1.9 & -2.0 & -1.8 & -1.7 & -1.4 \\
\hline [OIII] $\lambda 5007 / \mathrm{H} \beta^{a}$ & $3.52 \pm 6 \%$ & -5.4 & -9.5 & -4.6 & -0.9 & +0.8 & -1.1 & +1.8 \\
\hline \multicolumn{9}{|l|}{ Geometric corrections } \\
\hline$A$ & & & & 1.07 & 1.07 & 1.40 & 1.75 & 1.35 \\
\hline K & & & & 1.48 & 1.48 & 0.52 & 0.52 & 0.12 \\
\hline$B$ & & & & 0.58 & 0.58 & 1.45 & 3.31 & 1.15 \\
\hline
\end{tabular}

It seems clear that a more complex geometry for the gas is required in the models. Indeed, a complex density distribution is expected to change the ionization structure of the nebula, hence affecting the excitation diagnostics. Such a change would also modify the distribution of the main cooling ions (e.g., $\mathrm{O}^{++}$) and their efficiency to radiate energy away from the nebula. This may produce spatial temperature variations that are larger than in the case of a uniform density nebula.

\section{Models with constrained density distribution}

We used the well-observed distributions of the $\mathrm{H} \beta$, [OII] $\lambda 372(6+9)$ and [OIII] $\lambda 5007$ flux profiles along the optical slit and the $\mathrm{H} \alpha$ and [OIII] $\lambda 5007$ images to build a model of the gas density distribution. This model was used in all the photoionization models that follow.

\subsection{Construction of the density model}

\subsection{1. rms density assuming spherical symmetry}

In a nebula, the $\mathrm{H} \beta$ flux observed in a column of solid angle $\Omega$ and coordinates $(x, y)$ is:

$F_{\beta}(x, y)=\frac{\Omega}{4 \pi} \int_{Z_{1}(x, y)}^{Z_{2}(x, y)}\left(\varepsilon_{\beta} h v_{\beta} N_{\mathrm{e}} N_{\mathrm{p}} \epsilon\right)(x, y, z) \mathrm{d} z$

where $z$ is the coordinate along the line of sight, $\varepsilon_{\beta} h v_{\beta}$, the $\mathrm{H} \beta$ emissivity, and $N_{\mathrm{e}}$ and $N_{\mathrm{p}}$, the electron and proton density. Hence, assuming a given symmetry of the nebula, a model of 
Table 6. Parameters of the model density distribution, fitted on the slit $\mathrm{H} \beta$ profile and on the image. The distances are expressed in $10^{20} \mathrm{~cm}$, and the densities in $\mathrm{cm}^{-3}$. ${ }^{a}$ Projected minor and major semi-axes. ${ }^{b}$ Projected distance to the source.

\begin{tabular}{lll}
\hline \hline Parameter & Slit value & Image value \\
\hline$R_{1}$ & 1.01 & $0.97 \times 1.61^{a}$ \\
$R_{2}$ & 1.24 & $1.19 \times 1.98^{a}$ \\
$R_{3}$ & 2.49 & $2.39 \times 3.99^{a}$ \\
$N_{1}$ & 4.81 & 6.13 \\
$N_{2}$ & 9.13 & 10.9 \\
$N_{3}$ & 5.09 & 6.04 \\
$R_{G}^{b}$ & 0.40 & 0.40 \\
$\sigma_{G}$ & 0.17 & 0.17 \\
$N_{G}$ & 40.3 & 40.3 \\
\hline
\end{tabular}

the gas density distribution can be derived from the projected $F_{\beta}(x, y)$ distribution. This is what we did for NGC 588, from the flux observed in the CAHA slit and from the $\mathrm{H} \alpha$ image.

The $\mathrm{H} \alpha$ image suggests that the nebula is formed mainly by a bubble, possibly containing tenuous gas and surrounded by a halo. It also contains a bright knot located at the extremity of a filament. This knot and the other extremity of the filament are covered by the slit. With these considerations, we derived the following rms density $N_{\mathrm{H}} \sqrt{\epsilon}=f(x, z)$ in the plane covered by the optical slit, from the long-slit profile $F_{\beta}(x)$ :

- two "half-disks" located on either side of a central line of sight (of abscissa $x=0$ ), each composed of a density plateau with $f(x, z)=N_{1}$ for $r=\sqrt{x^{2}+z^{2}}<R_{1}$, an annulus with $f(x, z)=N_{2}>N_{1}$ for $R_{1} \leq r<R_{2}$; and a fading halo with $f(x, z)=N_{3}\left(R_{2} / r\right)^{2}$ for $R_{2} \leq r<R_{3}$

- two Gaussian round "blobs" on either side of the central line of sight, with the form $f(x, z)=N_{G} \exp \left(-\left(\left(x-x_{G}\right)^{2}+\right.\right.$ $\left.\left.\left(z-z_{G}\right)^{2}\right) /\left(2 \sigma_{G}^{2}\right)\right)$.

This model is presented in Fig. 9, where the good agreement between the observed $F_{\beta}(x)$ profile and the one predicted from the density model can be appraised. In the model that we fitted, the values of $N_{1}, N_{2}, N_{3}$ and $R_{3}$ depend on the considered side of the central line of sight $(x<0$ or $x>0)$. We simplified this by adopting average values of those parameters in what follows; these values are listed in the 2nd column of Table 6 . We also neglected the smaller Gaussian blob, because of its small contribution to the nebular flux $(3 \%$ of the $\mathrm{H} \beta$ flux in the slit). Furthermore, we adopted a distance of $4 \times 10^{19} \mathrm{~cm}$ between the bright blob and the stellar source, equal to the projected distance between this blob and the main condensation of the stellar cluster. In what follows, we will call $S$ the gas distribution that symmetrically surrounds the source, and $G$ the bright Gaussian knot. $G$ contributes to $30 \%$ of the $\mathrm{H} \beta$ flux seen through the optical slit and cannot be neglected.

We extended the model of density distribution to the case of the whole nebula, by using the $\mathrm{H} \alpha$ image (Fig. 1). For this, we stretched the spherical distribution $S$ in the image plane, according to the elliptical shape of the bubble in the image, while conserving the original dimensions along the line of sight. We then re-fitted $N_{1}, N_{2}$ and $N_{3}$ on the parts of the H $\alpha$ image unoccupied by the bright central filament. The values obtained,

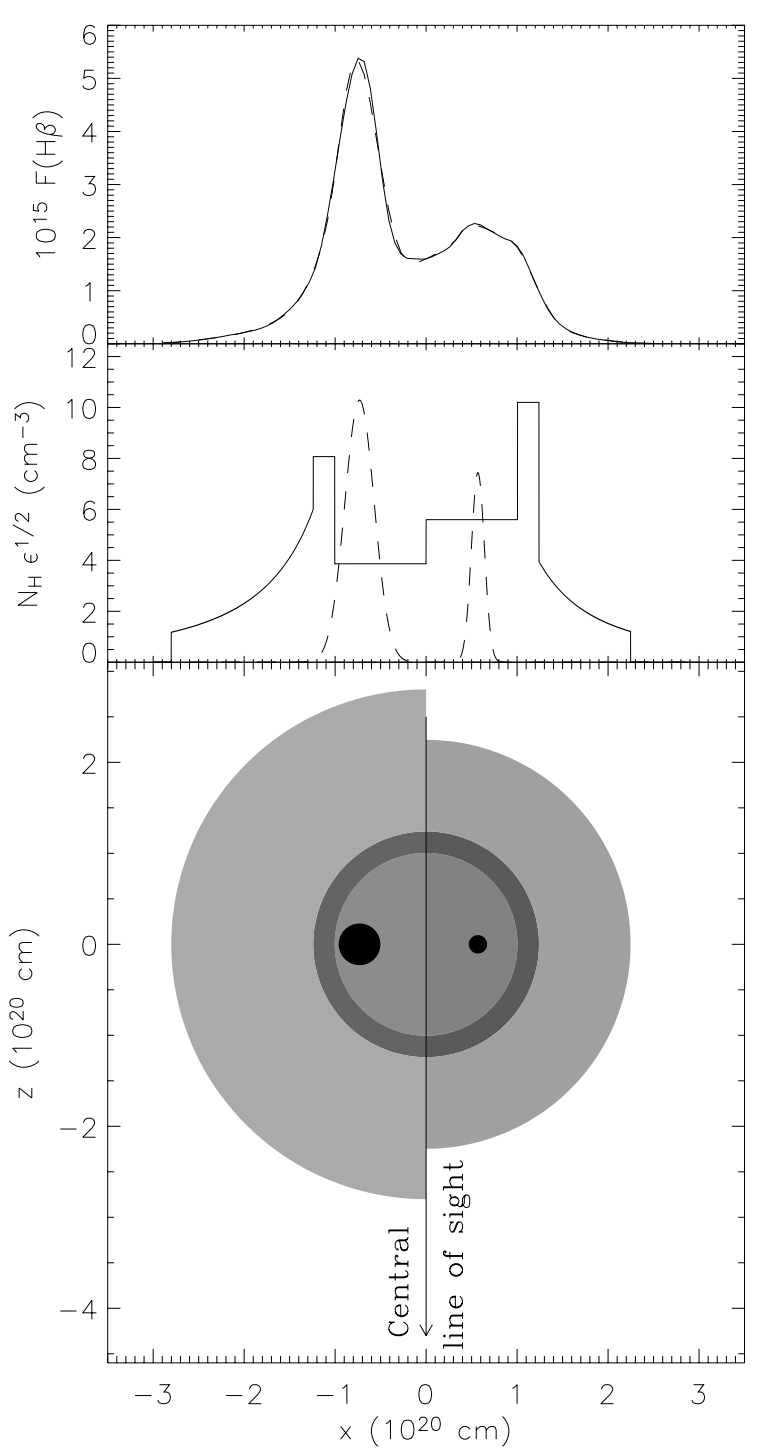

Fig. 9. Upper panel: $\mathrm{H} \beta$ long-slit profile (full line: observed; dashed line: model). Middle panel: model of the $N_{\mathrm{H}} \sqrt{\epsilon}$ distribution (full line: gas surrounding the source; dashed lines: Gaussian blobs). In this panel, the value of $N_{\mathrm{H}} \sqrt{\epsilon}$ for the two Gaussian blobs was divided by 4 , for better visibility. Lower panel: schematic view of the model density profile in the plane section of the optical slit. The $x$ axis is parallel to the slit, while the $z$ axis is parallel to the line of sight. The two blobs (represented by black dots) may actually be located at any position along $z$.

listed in Table 6 , are slightly different from the ones obtained for the optical slit. $G$ contributes to $\approx 7 \%$ of the total $\mathrm{H} \beta$ flux of the nebula.

The knot $G$ is relatively confined and is not centered on the source. Since our code only processes spherically symmetric gas distributions, we modeled $G$ with a shell of Gaussian density profile, but we integrated the line fluxes in a way adapted to its non-radial gas distribution.

\subsubsection{Accounting for non-sphericity}

The model presented above assumes spherical symmetry of its different components, in contradiction with what stands out of 

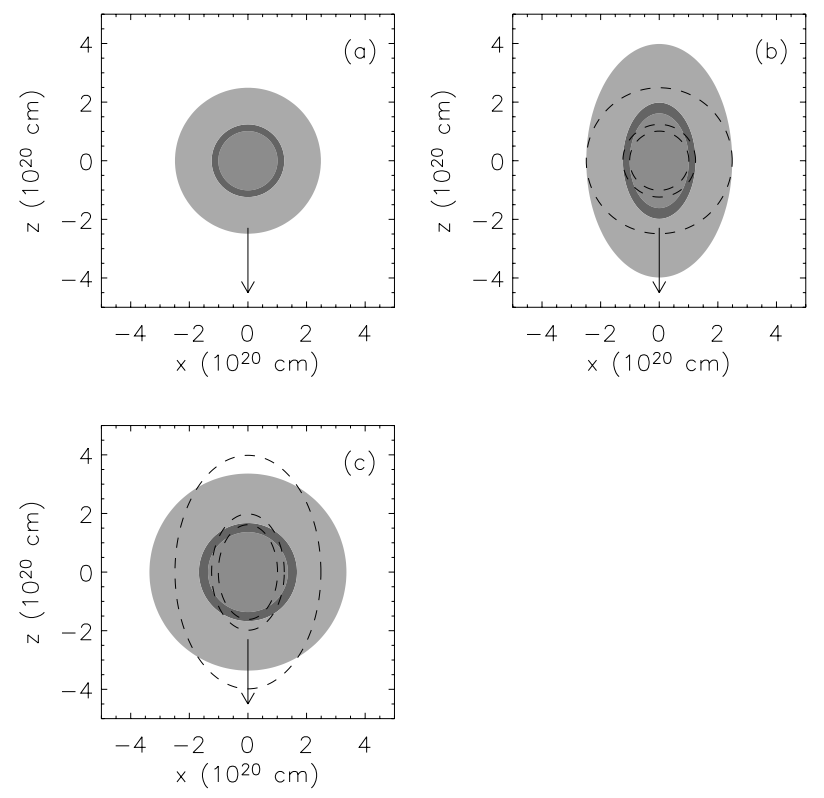

Fig. 10. Illustration of the geometrical correction for the gas distribution of $S$. The arrows indicate the direction of the observer. a) Model inferred in Sect. 6.1.1. b) Possible shape of the real distribution. c) Approximation of $\mathbf{b}$ ) adopted in photoionization models in Sects. 6-7.

the $\mathrm{H} \alpha$ image. Indeed, the projected images of both $S$ and $G$ are elliptical, showing that their gas distributions are not isotropic. Furthermore, such asymmetry can exist along the line of sight with respect to the plane of the sky and it that case, it remains undetectable because of the projection of the signal received from the nebula.

Our photoionization code assumes spherical symmetry, meaning that the accurate geometry of $S$ cannot be processed. This is why we decided to model $S$ with a spherical gas distribution obtained by homothetic transform of the three-component distribution $N_{\mathrm{H}} \epsilon=f(r)$ established in Sect. 6.1.1. This transform, illustrated in Fig. 10, is meant to infer sizes to the different structures (e.g., the bubble) that are averages of the real dimensions of these structures. We used two homothetic factors, $A$ for the lines seen through the optical slit and $B$ for the lines integrated over the whole nebula. $A$ and $B$ are basically different, because they are representative of different portions of the nebula.

In Sect. 6.1.1, we assumed $G$ to be spherical. Nevertheless, it may be stretched or squeezed along the line of sight with respect to the plane of the sky. In this case, the volume of $G$ is not the one that we inferred in Sect. 6.1.1. Since its $\mathrm{H} \beta$ flux is fixed, its density is also different from the one initially inferred. This effect must be taken into account, because of the importance of the density on the ionization state of the gas. Hence, we introduced a factor $K$ of stretching $G$ along the line of sight with respect to the projected view.

The geometric corrections that we introduced have significant consequences for the ionization state predicted by the models. In particular, they modify the [OIII] $\lambda 5007 /[\mathrm{OII}]$ $\lambda 372(6+9)$ and [OIII] $\lambda 5007 / \mathrm{H} \beta$ ratios in both $S$ and $G$. We used those ratios to constrain the coefficients $A, B$ and $K$, following the procedure explained in Appendix B.

\subsection{Model results}

Model [DD1] of Table 5 was established with a filling factor of $\epsilon=1$. All the parameters other than the density are the same as those of model [FS] and [HB] (Sect. 5). While $T_{\mathrm{IR}}(\mathrm{OIII})$ is marginally reproduced, $T_{\text {opt }}(\mathrm{OIII})$ is still much underestimated.

In order to attempt to fit the optical temperature diagnostics - and temporarily ignoring the optical/IR diagnostic - we modified the input carbon and sulfur abundances, since these two elements are the most efficient coolants among those whose abundances were not directly measured. The other parameters, including $A, K$ and $B$, were unchanged. We were able to fit the temperature diagnostics only with very small carbon and sulfur abundances: even if the latter are set to $1 / 10$ of the original values, $T_{\text {opt }}(\mathrm{OIII})$ and $T(\mathrm{SII})$ are almost too small compared to the observed ones (column [DD2] of Table 5). Such small abundances are physically unlikely, so we rejected the model.

\section{Further refining of the models}

\subsection{Using the degree of freedom on the stellar ionizing spectrum}

So far, we only used the 4.2 Myr cluster model of Jamet et al. (2004), which corresponds to their best fit model. However, acceptable cluster ages range from 3.6 to 4.4 Myr. The hardness of the Lyman continuum decreases with increasing age. Therefore, we used the cluster spectrum corresponding to 3.6 Myr to test whether it is at least able to explain the $11000 \mathrm{~K}$ temperature diagnosed by the optical lines (ignoring again the optical/IR diagnostic).

We discarded the filling factor $\epsilon=1$, because it requires values of $A$ and $B$ that are too large, which is equivalent to assuming that the nebula is extremely stretched along the line of sight. For $\epsilon=0.1$ and using the original carbon and sulfur abundances, $T_{\text {opt }}(\mathrm{OIII})$ is still underestimated. However, this issue is resolved if the sulfur abundance is divided by 2 (model [DDS]), a value that can be considered acceptable.

Although using an ionizing spectrum harder than the one first used improves the prediction of the optical temperature diagnostics, the large temperature discrepancy between the latter and $T_{\mathrm{IR}}(\mathrm{OIII})$ remains unexplained.

\subsection{Appending local density fluctuations}

Most GHRs present complex local structures, such as filaments and condensations (e.g., NGC 604 and 30 Dor: see, respectively, Maíz-Apellániz et al. 2004; Walborn et al. 2002). Such structures may exist in NGC 588 and remain invisible in our ground-based images due to insufficient angular resolution. Local structures in the gas are usually modeled by using filling factors $\epsilon<1$, i.e. assuming that the gas is entirely condensed in uniform clumps occupying a fraction $\epsilon$ of the available volume. However, it is more realistic to assume that the density varies 
continuously, being higher in the bright structures than in the regions situated between them.

A continuous density distribution with non-zero density between the bright structures may increase the amplitude of the spatial temperature variations since, due to a different distribution of the cooling ions, the temperature in the diffuse component is expected to be different from that in the filaments.

\subsubsection{Formalism of the fluctuations}

The rms density of Sect. 6 was multiplied by the following periodic radial function:

$s\left(r ; H, r_{0}\right)=\frac{L+(1-L)\left|\cos \left(\pi r / r_{0}\right)\right|^{n}}{\sqrt{H}}$

where $L=\min (s) / \max (s)$ defines a diffuse gas component between a series of clumps, whose condensation level is described by the parameter $n ; H$ is a normalization factor such that $\left\langle f^{2}\right\rangle_{r}=1$. $H$ ensures that the observed $\mathrm{H} \beta$ flux distribution will not be modified within scales larger than $r_{0}$ since, locally, the $\mathrm{H} \beta$ emission is proportional to $s(r)^{2}$. We note that $\max \left(s^{2}\right)=1 / H$. Furthermore, $H$ only depends on $L$ and $n$, and the actual value $r_{0}$ is only required to be small, in order to simulate small-scale gas structures. Finally, we will deal not with $L$, but with $\phi=L^{2} / H$, the fractional contribution of the diffuse component to the $\mathrm{H} \beta$ flux. We applied the density fluctuations only to $S$, and kept the original smooth density profile for $G$.

\subsubsection{Results}

We computed a few models with various values of $H$ and $\phi$, using the stellar spectrum for 3.6 Myr and the standard elemental abundances. The different models gave similar results. The one shown in Table 5 ([DF]) was obtained for $H=0.1$ and $\phi=0.2$. It can be seen that the fluctuations of density do not resolve the discrepancy between $T_{\text {opt }}(\mathrm{OIII})$ and $T_{\mathrm{IR}}(\mathrm{OIII})$.

Figure 11 shows the radial distribution of $N_{\mathrm{H}}, T_{\mathrm{e}}, \mathrm{O}^{+} / \mathrm{O}$, $\mathrm{O}^{++} / \mathrm{O}$ and $\mathrm{H}^{0} / \mathrm{H}$ in $S$. The variations caused by the density fluctuations to the ionization state induce variations in the cooling rate of the different chemical elements. In particular, in the outer parts of the nebula, neutral hydrogen becomes an efficient cooling agent by collisional excitation of Ly $\alpha$. Unfortunately, the resulting variations of temperature between the density clumps and the surrounding gaps are smaller than $500 \mathrm{~K}$.

Close to the source, the temperature fluctuations associated to the clumps are due in large part to the ionization structure of sulfur. Consequently, we multiplied the sulfur abundance by 2 and checked the changes in the temperature distribution. We found that this distribution was modified very little, so we concluded that density fluctuations are not a solution to the $T_{\text {opt }}(\mathrm{OIII})-T_{\mathrm{IR}}(\mathrm{OIII})$ conflict.

\subsection{Models with dust}

The effects of dust in the thermal balance of planetary nebulae were studied by Stasińska \& Szczerba (2001) with detailed models. They show that dust grains provide additional heating by photoelectric emission, proportional to the dust-to-hydrogen

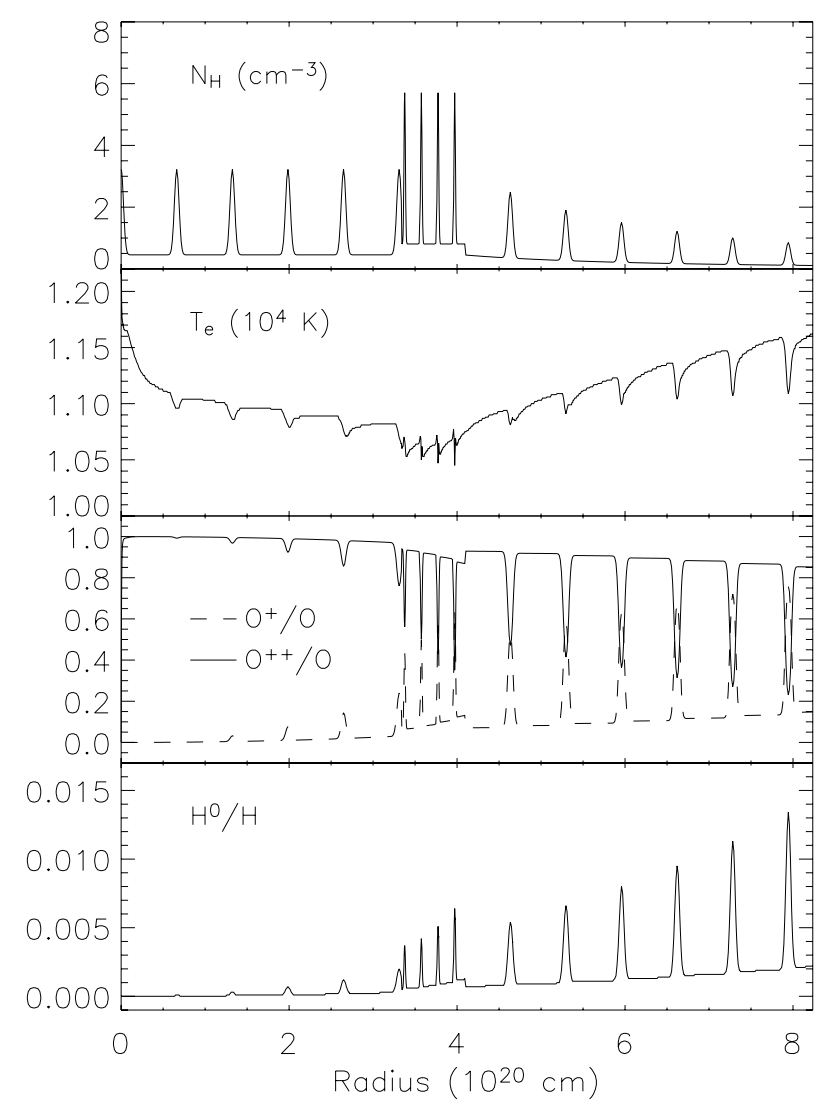

Fig. 11. Radial profiles of $N_{\mathrm{H}}, T_{\mathrm{e}}$, and the relative abundances of the most coolant ions, in model [DF].

mass density ratio $\rho_{\mathrm{d}} / \rho_{\mathrm{H}}$ and to the local ionization parameter $U(r)=Q\left(\mathrm{H}^{0}\right) /\left(4 \pi r^{2} N_{\mathrm{e}} c\right)$. Hence, in a nebula where the dust density and the average ionization parameter are large enough, the decrease in $U(r)$ with distance $r$ to the source causes a significant negative gradient of the temperature as a function of $r$. Local density fluctuations also change the temperature significantly toward lower values in clumps due to a smaller value of $U(r)$ in them.

We adopted the grain size distribution of Mathis et al. (1977) for half the total grain mass and a small grain population (with grain sizes $<0.01 \mu \mathrm{m}$ ) for the other half mass. We limited our study of dust grain effects to the case of the cluster spectrum for 3.6 Myr, which gives the highest value of $Q\left(\mathrm{H}^{0}\right)$ among our set of cluster spectra. This allowed us to increase $U(r)$ as much as possible. It also allowed us to maximize the threshold of $\rho_{\mathrm{d}} / \rho_{\mathrm{H}}$ above which dust grains would absorb too large a fraction of the ionizing photons and cause the ionization bound to be closer to the source than the observed bound. This threshold is $\rho_{\mathrm{d}} / \rho_{\mathrm{H}}=5 \times 10^{-4}$ for the adopted ionizing stellar spectrum.

We adopted $H=0.01$ and $\phi=0.5$ for the density fluctuation function. With these parameters, the diffuse gas and the clumps contribute equally to the $\mathrm{H} \beta$ flux and $U(r)$, which is inversely proportional to $s(r)$, changes by a factor as large as $1 / \sqrt{\phi H}=14$ between the maxima of the clumps and the diffuse gas component.

Column [DG] of Table 5 shows the line ratios predicted with $\rho_{\mathrm{d}} / \rho_{\mathrm{H}}=5 \times 10^{-4}$. Even with such a high dust-to-gas 


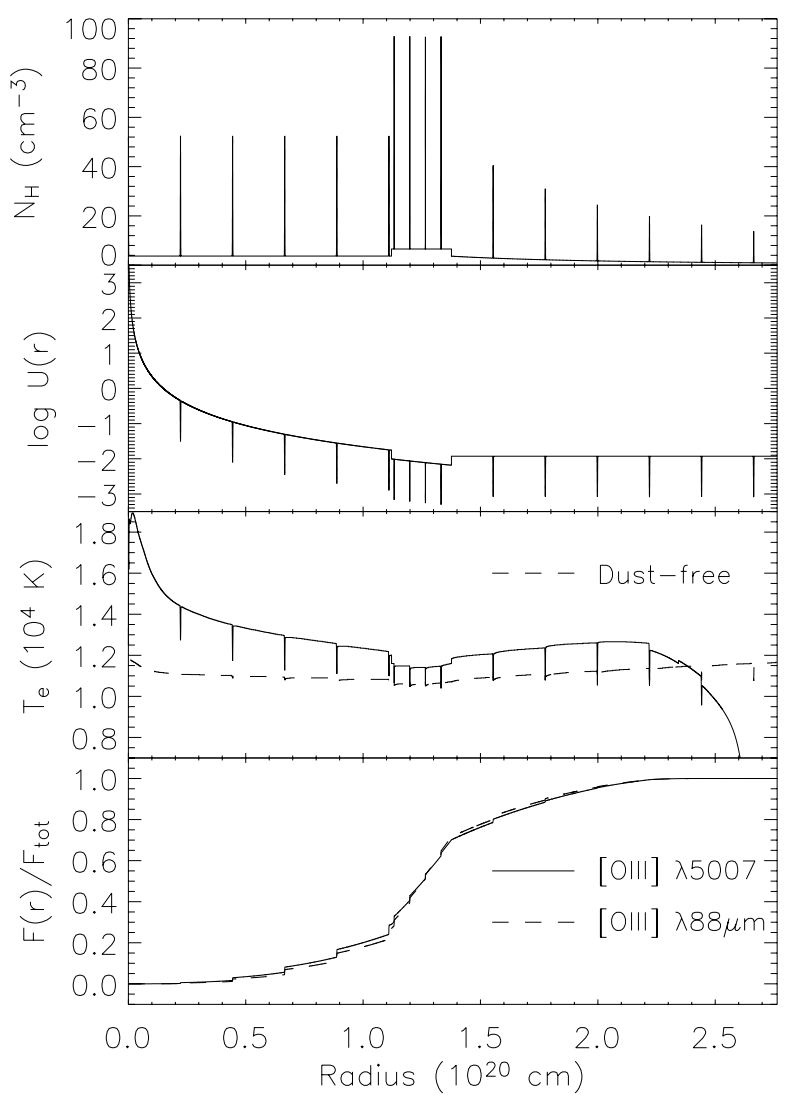

Fig. 12. Radial profiles of $N_{\mathrm{H}}, U, T_{\mathrm{e}}$ (three upper panels), and fractional [OIII] $\lambda 5007$ and [OIII] $\lambda 88 \mu \mathrm{m}$ fluxes integrated through increasing circular apertures (lower panel), in model [DG].

density ratio, we were unable to obtain temperature fluctuations large enough to explain the conflict between $T_{\text {opt }}(\mathrm{OIII})$ and $T_{\mathrm{IR}}(\mathrm{OIII})$ and both temperatures are largely overestimated. Indeed, in addition to a global increase of temperature, the three effects of dust on the thermal balance are a significant steepening of the temperature in a small zone close to the source, which only contributes to $\approx 10 \%$ of the [OIII] $\lambda 5007$ and [OIII] $\lambda 88 \mu \mathrm{m}$ fluxes, a drop of the temperature in the faint outskirts of the nebula and a drop of the temperature in the clumps by up to $\approx 2000 \mathrm{~K}$, which is insufficient to explain the temperature discrepancy between the observed diagnostics. This is illustrated in Fig. 12, where the radial profiles of $N_{\mathrm{H}}$, $T_{\mathrm{e}}$, and $U(r)$ are plotted together with the fractional fluxes of the [OIII] $\lambda 5007$ and [OIII] $\lambda 88 \mu \mathrm{m}$ integrated through circular apertures whose radii are given by the abscissa axis.

To reproduce at least $T_{\mathrm{opt}}(\mathrm{OIII}), \rho_{\mathrm{d}} / \rho_{\mathrm{H}}$ would have to be smaller than in model [DG]. In that case, by being smaller than in the latter, the amplitude of the temperature variations would be even further from accounting for the $T_{\text {opt }}(\mathrm{OIII})-T_{\mathrm{IR}}(\mathrm{OIII})$ discrepancy. Dust grains are therefore an unsatisfactory solution.

\subsection{Spatial distribution of the stars}

It is well known that in a specific point of a nebula, the energy gain provided by photoionization mainly depends of the

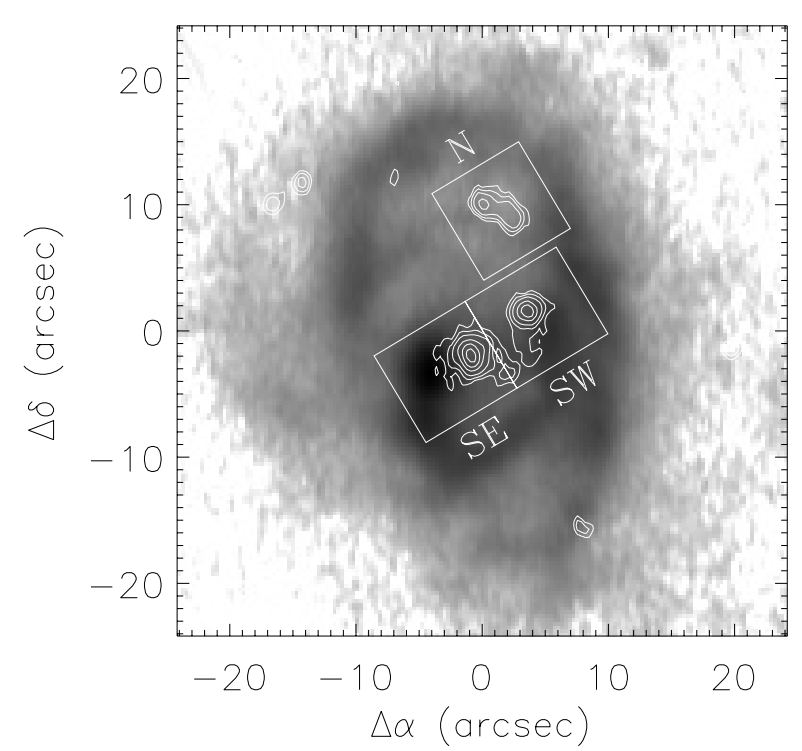

Fig. 13. Zones of extraction of spectra in Sect. 7.4.

Table 7. Some spectral properties of the three zones N, SW and SE and of the whole cluster, assuming an age of 3.6 Myr. $Q_{\text {tot }}\left(\mathrm{H}^{0}\right)$ is the total photon luminosity of the cluster in the Lyman continuum range.

\begin{tabular}{lll}
\hline \hline Zone & $Q\left(\mathrm{H}^{0}\right) / Q_{\text {tot }}\left(\mathrm{H}^{0}\right)$ & $T_{*}(\mathrm{kK})$ \\
\hline $\mathrm{N}$ & $25 \%$ & 34 \\
$\mathrm{SW}$ & $26 \%$ & 38 \\
$\mathrm{SE}$ & $49 \%$ & 48 \\
All & $100 \%$ & 41 \\
\hline
\end{tabular}

hardness of the local Lyman continuum (e.g., Stasińska 2004), and in particular on the temperature $T_{*}$ given by:

$\frac{3}{2} k T_{*}=\frac{\int_{v_{0}}^{+\infty}\left(1-v_{0} / v\right) F_{v} \sigma_{v} \mathrm{~d} v}{\int_{v_{0}}^{+\infty} F_{v} \sigma_{v} /(h v) \mathrm{d} v}$

where $F_{v}$ is the flux received from the stars, $h v_{0}$ the hydrogen ionization threshold, and $\sigma_{v}$ the hydrogen ionization cross-section. In the case of NGC 588, the ionizing stars are distributed on spatial scales comparable to the size of the nebula, and one may expect the Lyman continuum received to vary from point to point, as it is harder close to hotter stars. In that case, the temperature structure of the gas is sensitive to the distribution of the stars.

To evaluate the impact of the heterogeneity of the cluster on the temperature structure of the gas, we divided the cluster into three zones, indicated in Fig. 13, and extracted the stellar spectrum of each of them, adopting the age of 3.6 Myr for the cluster. As shown in Table 7, the three zones individually produce a significant fraction of the ionizing photons and have different temperatures $T_{*}$. Altogether they provide all the ionizing flux of the cluster. The zones SE, close to the $\operatorname{knot} G$, and SW, also covered by the optical slit, are dominated by the two Wolf-Rayet stars (WR) of the cluster, i.e. respectively stars \#2 and \#1 of Jamet et al. (2004). They are the brightest and hottest two stars of the cluster. Zone $\mathrm{N}$ is dominated by a group of three stars, much cooler and less luminous than the two WR. 
Table 8. Empirical temperatures yielded by the spectra of the zones $\mathrm{N}, \mathrm{SW}$ and SE and of the whole cluster.

\begin{tabular}{llllll}
\hline \hline zone & $T_{\text {opt }}(\mathrm{OIII})$ & $T(\mathrm{OII})$ & $T(\mathrm{SII})$ & $T(\mathrm{NII})$ & $T_{\mathrm{IR}}(\mathrm{OIII})$ \\
\hline $\mathrm{N}$ & 9990 & 9860 & 7960 & 10090 & 9580 \\
$\mathrm{SW}$ & 10220 & 10080 & 8070 & 10300 & 9760 \\
$\mathrm{SE}$ & 11610 & 11450 & 8830 & 11610 & 10880 \\
All & 10840 & 10740 & 8430 & 10920 & 10250 \\
\hline
\end{tabular}

We re-computed the model [DDS] of Sect. 7.1, but readopted the original sulfur abundance, and successively replaced the spectrum of the cluster by the ones of the three zones N, SW, and SE, each rescaled to the ionizing rate of the whole cluster. The results are shown in Table 8, in terms of empirical temperatures at $N_{\mathrm{e}}=70 \mathrm{~cm}^{-3}$. While the spectra of $\mathrm{N}$ and SW result in very similar temperatures of the gas, the one of SE yields temperatures higher than the latter by $\approx 1500 \mathrm{~K}$. Thus, close to the stars of the zone SE, the gas temperature may undergo a significant excess with respect to the overall nebula. This is the case of $G$, in particular, much closer to SE than to the other parts of the cluster, at least in the projected sight of the nebula. Hence, we may a priori suspect $G$ of being responsible for an increase in $T_{\text {opt }}(\mathrm{OIII})$, since the latter is measured through the optical slit, where $G$ provides $\approx 40 \%$ of the [OIII] $\lambda 5007$ flux. However, by replacing the model of $G$ inferred from the total cluster spectrum with the one derived from the spectrum of WE, we oberved an increase of $T_{\text {opt }}(\mathrm{OIII})$ by $300 \mathrm{~K}$ only.

The spatial structure of the cluster of NGC 588 turns out not to provide a satisfactory explanation of the $T_{\mathrm{opt}}(\mathrm{OIII})-T_{\mathrm{IR}}(\mathrm{OIII})$ conflict that we encountered.

\section{Sources of energy other than the ionizing flux}

\subsection{Shocks}

A hypothesis that is often advocated to explain the large temperature inhomogeneities observed in some GHRs is the supply of energy in the form of shocks resulting from the winds of massive stars and from supernovae. Those shocks are expected to raise the temperature abruptly in thin slabs of gas. However, alteration of the thermal structure induced by shocks in nebulae has been studied little (see, however, the case of NGC 2363: Luridiana et al. 2001). A "hot-spot" model of this phenomenon has been proposed by Binette \& Luridiana (2000), which we applied it to NGC 588.

No supernova remnant was identified within NGC 588 either in the X-ray survey of Pietsch et al. (2004) or in the radiooptical one of Gordon et al. (1999). We therefore assumed that the kinetic power released by the stellar cluster is conveyed only by winds. Using the theoretical wind powers implemented in Starburst99 (Leitherer et al. 1999), we found that the stars of NGC 588 release a kinetic power $\dot{E}=1.1 \times 10^{38} \mathrm{erg} \mathrm{s}^{-1}$, close to the $\mathrm{H} \beta$ luminosity of the nebula. On the other hand, the photoionization gain of the nebula is 15-20 times the $\mathrm{H} \beta$ luminosity. As a result, if we assume that all the energy of the shocks enhances the collisionally excited lines of the nebula, the term $\Gamma_{\text {heat }}$ of Binette \& Luridiana (2000) is 0.05-007. Given the low metallicity of the nebula, this leads to temperature fluctuations below $t^{2}=0.01$. This is clearly insufficient to resolve the $T_{\text {opt }}(\mathrm{OIII})-T_{\mathrm{IR}}(\mathrm{OIII})$ conflict. To reach $t^{2}=0.087$, the kinetic power of the stellar winds would have to be at least 5 times larger than that computed.

\subsection{Electron conduction from hotter plasma layers}

Hot plasma slabs generated by shocks in a nebula are expected to convey heat to the surrounding gas by electron conduction, creating transition zones between those slabs and the gas excited by photoionization alone. Maciejewski et al. (1996) constructed a model nebula consisting of gas sheets, each of width $\mathcal{H}$, separated by thin layers of very hot and tenuous gas. They studied the thermal structure of the sheets undergoing heat conduction by electrons, and showed that this structure is able to enhance the [OIII] $\lambda 4363$ line, while lines such as $\mathrm{H} \beta$ and [OIII] $\lambda 5007$ are modified little. Through their Eqs. (9), (10), and (14)-(18), they suggest a relation between $\mathcal{H}$, the density $N_{\mathrm{p}}$, the temperature $T_{\text {eq }}$ in zones undergoing photoionization alone, and the temperature diagnostic $T_{\text {opt }}(\mathrm{OIII})$. Adopting $T_{\text {eq }}=8000 \mathrm{~K}$ and ignoring Eq. (18), we found $\mathcal{H} \sim 10^{15}$ $10^{16} \mathrm{~cm}$, within the range of densities $N_{\mathrm{p}}$ inferred in Sect. 6.1.1. This estimate is of course rough, because it does not accurately take into account the density structure of the nebula (smooth or with clumps), the ionization state of the gas, and the real value of $T_{\text {eq. }}$. However, given the size of the nebula, our estimate of $\mathcal{H}$ holds the presence of $\sim 10^{4}-10^{5}$ layers of very hot plasma; even with higher values of $\mathcal{H}$, the number of those layers would be much too large to be realistic. We can therefore reject the electron conduction of heat as an explanation of the large difference between $T_{\text {opt }}(\mathrm{OIII})$ and $T_{\mathrm{IR}}(\mathrm{OIII})$.

\section{Uncertainties in $\mathrm{O} / \mathrm{H}$ and $\mathrm{Ne} / \mathrm{O}$}

Since the thermal balance of NGC 588 is not fully understood, the estimates of abundance ratios, either empirical or obtained with models, are uncertain not only through the line ratios that are used to probe them, but also through the discrepancy that exists between the different temperature diagnostics. We estimated this uncertainty for the $\mathrm{O}^{++} / \mathrm{H}$ and $\mathrm{Ne}^{++} / \mathrm{O}^{++}$abundance ratios, as $\mathrm{O}^{++}$and $\mathrm{Ne}^{++}$are the two ions for which we have observations in both optical and IR ranges. We compared the empirical $\mathrm{O}^{++} / \mathrm{H}$ and $\mathrm{Ne}^{++} / \mathrm{O}^{++}$ratios obtained by using the longslit values of [OIII] $\lambda 5007 / \mathrm{H} \beta$, [NeIII] $\lambda 3869 /[\mathrm{OIII}] \lambda 5007$, and $T_{\mathrm{opt}}(\mathrm{OIII})$ with those derived from the integrated values of [OIII] $\lambda 5007 / \mathrm{H} \beta$, [NeIII] $\lambda 16 \mu \mathrm{m} /[\mathrm{OIII}] \lambda 88 \mu \mathrm{m}$, and $T_{\mathrm{IR}}$ (OIII).

The results are reported in Table 9 . It can be seen that the computed $\mathrm{O}^{++} / \mathrm{H}$ abundance ratio depends a lot on the selected temperature diagnostic and that the actual value of $\mathrm{O}^{++} / \mathrm{H}$ is known to within \pm 0.2 dex. Since $\mathrm{O}^{++}$is the dominant ion of $\mathrm{O}$ in the nebula, the elemental abundance ratio $\mathrm{O} / \mathrm{H}$ is uncertain by a similar amount. Assuming that the empirical $\mathrm{O} / \mathrm{O}^{++}$ derived from the optical slit data is correct $\left(\mathrm{O} / \mathrm{O}^{++}=1.39\right)$, and depending on the adopted [OIII] temperature, $\mathrm{O} / \mathrm{H}$ ranges approximately from $1.5 \times 10^{-4}$ to $3.6 \times 10^{-4}$. The two estimates of the $\mathrm{Ne}^{++} / \mathrm{O}^{++}$ratio, to which $\mathrm{Ne} / \mathrm{O}$ is expected to be close, differ by a smaller factor of 1.6. 
Table 9. $\mathrm{O}^{++} / \mathrm{H}^{+}$and $\mathrm{Ne}^{++} / \mathrm{O}^{++}$abundance ratios derived from different diagnostics. The error bars include the uncertainties related to the temperature diagnostics and to the $[\mathrm{OIII}] / \mathrm{H} \beta$ and $[\mathrm{NeIII}] /[\mathrm{OIII}]$ line ratios that we used.

\begin{tabular}{llll}
\hline \hline Set of data & Temperature & Line ratio & Abundance ratio \\
\hline $\mathrm{O}^{++} / \mathrm{H}^{+}$ & & & \\
Optical slit & $T_{\text {opt }}(\mathrm{OIII})=1140 \pm 180 \mathrm{~K}$ & {$[\mathrm{OIII}] \lambda 5007 / \mathrm{H} \beta=4.05 \pm 0.18$} & $(1.04 \pm 0.07) \times 10^{-4}$ \\
Integrated & $T_{\mathrm{IR}}(\mathrm{OIII})=8420 \pm 690 \mathrm{~K}$ & {$[\mathrm{OIII}] \lambda 5007 / \mathrm{H} \beta=3.52 \pm 0.20$} & $(2.55 \pm 0.72) \times 10^{-4}$ \\
\hline $\mathrm{Ne}^{++} / \mathrm{O}^{++}$ & & & \\
Optical slit & $T_{\text {opt }}(\mathrm{OIII})=1140 \pm 180 \mathrm{~K}$ & {$[\mathrm{NeIII}] \lambda 3869 /[\mathrm{OIII}] \lambda 5007=0.0911 \pm 0.0047$} & $0.257 \pm 0.014$ \\
Integrated & $T_{\mathrm{IR}}(\mathrm{OIII})=8420 \pm 690 \mathrm{~K}$ & {$[\mathrm{NeIII}] \lambda 16 \mu \mathrm{m} /[\mathrm{OIII}] \lambda 88 \mu \mathrm{m}=0.257 \pm 0.085$} & $0.164 \pm 0.059$ \\
\hline
\end{tabular}

It is interesting to note that the value of $\mathrm{Ne}^{++} / \mathrm{O}^{++}$derived from $T_{\mathrm{IR}}(\mathrm{OIII})$ and [NeIII] $\lambda 16 \mu \mathrm{m} /[\mathrm{OIII}] \lambda 88 \mu \mathrm{m}$ closely matches the solar $\mathrm{Ne} / \mathrm{O}$ ratio recommended by Lodders (2003), which is 0.15 . This agrees with the usual idea that $\mathrm{Ne} / \mathrm{O}$ is close to solar in environments such as GHRs (e.g., Garnett 2004). Furthermore, the $\mathrm{O} / \mathrm{H}$ estimate derived from $T_{\mathrm{IR}}(\mathrm{OIII})$ and the integrated [OIII] $\lambda 5007 / \mathrm{H} \beta$ ratio is found to be close to the average one of supergiant stars of M 33 situated at similar galactocentric distances $\left(\mathrm{O} / \mathrm{H} \approx 3 \times 10^{-4}\right.$ : Urbaneja et al. 2005). Hence, the integrated optical/IR line ratios suggest "standard" abundances in the nebula. On the contrary, the optical spectrum suggests peculiar abundances with a $\sim 50 \% \mathrm{O} / \mathrm{H}$ depletion and a $\mathrm{Ne} / \mathrm{O}$ excess of $\sim 70 \%$.

\section{Conclusion}

This work was motivated by the frequent use of giant HII regions to determine the chemical composition of the interstellar medium in galaxies. The usual methods are simple in principle but need to be validated by realistic models.

We have carried out a comprehensive study of the giant HII region NGC 588. We gathered and carefully combined a large number of observations consisting of spectroscopic data in the UV, visible, and infra-red domains, as well as of narrowband images. The most outstanding property of this HII region is that the temperature derived from the $[\mathrm{OIII}] \lambda 5007 / \lambda 88 \mu \mathrm{m}$ ratio is lower by about $3000 \mathrm{~K}$ than the temperature obtained with four different optical temperature diagnostics. This suggests either the presence of large temperature inhomogeneities or the incorrectness of flux measurements. After a careful analysis of the observational data, we excluded this latter possibility, because it would imply that the [OIII] $\lambda 88 \mu \mathrm{m}$ line flux has been overestimated by a factor of 2 . We then constructed photoionization models with the aim of reproducing the observational data, including all the temperature diagnostics.

In a previous paper (Jamet et al. 2004), we conducted a detailed star-by-star analysis of the cluster responsible for the ionization of the HII region and obtained an ionizing spectral energy distribution based on the most up-to-date stellar atmosphere models. We used this spectral energy distribution as input to photoionization models tailored to reproduce all the observed spectral diagnostics. Our procedure takes the different apertures into account through which the spectroscopic data are obtained. We started with the simplest density structures, i.e. a homogeneous sphere and then a bubble. Indeed, most of our understanding of giant HII regions is based on such models.
Since these simple models failed to reproduce all the temperature diagnostics, we proceeded to more complex representations. Our strategy was to stick as closely as possible to the observational constraints provided by the spectra and images and to explore the free parameters left systematically. These include abundances of unconstrained elements such as carbon, density fluctuations, dust grains, spatial extent of the ionizing cluster, shock heating and conductive heating. No satisfactory solution was found. We stress that, although there are other cases of HII regions for which published photoionization models were not able to reproduce the observed temperatures, this is the first time that such a thorough analysis of a giant HII region has been performed and such a large number of observational constraints used.

The fact that no acceptable model has been found yet has important consequences. Two explanations can be advanced:

- Either the energy balance of this object is not understood. This then might also apply to giant HII regions in general (Esteban et al. 2002, and references therein). In that case, abundances derived from optical lines are not as accurate as usually thought, even in objects where temperature diagnostics are available.

- Or, the uncertainty in the [OIII] $\lambda 88 \mu$ m fluxes measured by ISO is larger than expected. If this is the case, results based on the analysis of this line, even in other contexts, should be regarded with caution.

For the time being, in the case of NGC 588, we can only say that the $\mathrm{O} / \mathrm{H}$ ratio probably lies somewhere between $\approx 1.5 \times 10^{-4}$ and $3.6 \times 10^{-4}$. However, we found that the $\mathrm{O} / \mathrm{H}$ and $\mathrm{Ne} / \mathrm{O}$ ratios derived from optical/IR integrated data are more consistent with at least some comparison sources (supergiants in M 33 for $\mathrm{O} / \mathrm{H}$, the Sun for $\mathrm{Ne} / \mathrm{O}$ ) than those derived from the optical spectrum and should probably be preferred to the latter.

Alternatively to temperature fluctuations, abundance inhomogeneities might be a solution to the conflict between the temperature diagnostics. They have been advocated in planetary nebulae to explain the inconsistencies between abundances derived from optical forbidden lines and from recombination lines (Liu 2003). Such conflicts are also observed in giant HII regions (e.g., Esteban et al. 2002), albeit to a lesser extent. Though abundance inhomogeneities are theoretically expected not to occur in HII regions (Tenorio-Tagle 1996), the current view is changing (Stasińska et al. 2005; Tsamis \& Péquignot 2005). A measurement of oxygen recombination line intensities in NGC 588 would provide an important constraint to 
investigating the presence and significance of abundance inhomogeneities in this object. In the case where there the latter is present, one needs to investigate the precise meaning of the chemical abundances derived with various techniques.

Acknowledgements. We are grateful to Ryszard Szczerba for precious help with the ISO data. We also thank Lise Deharveng, Ariane Lançon, Miguel Cerviño and Valentina Luridiana for very useful suggestions. Funding was provided by French CNRS Programme National GALAXIES, by Spanish grants AYA-2001-3939-C03-01, AYA-2001-2089, AYA2001-2147-C02-01 and AYA2004-2703, and by the French-Spanish bi-lateral program PICASSO/Acción Integrada HF2000-0143.

\section{References}

Auer, L., \& Mihalas, H. D. 1972, ApJS, 24, 193

Binette, L., \& Luridiana, V. 2000, RMxAA, 36, 43

Burstein, D., \& Heiles, C. 1984, ApJS, 53, 33

Esteban, C. 2002, Rev. Mex. Astron. Astrofis. Ser. Conf., 12, 56

Esteban, C., Peimbert, M., Torres-Peimbert, S., \& Rodríguez, M. 2002, ApJ, 581, 241

García-Vargas, M. L., González-Delgado, R. M., Pérez, E., et al. 1997, ApJ, 478, 112

Garnett, D., 2004, in Cosmochemistry. The melting pot of the elements. XIII Canary Islands Winter School of Astrophysics, Puerto de la Cruz, Tenerife, Spain, November 19-30, 2001, ed. C. Esteban, R. J. García López, A. Herrero, \& F. Sánchez, Cambridge contemporary astrophysics, 115

González Delgado, R. M., \& Pérez, E. 2000, MNRAS, 317, 64

Gordon, S. M., Duric, N., Kirshner, R. P., Goss, W. M., \& Viallefond, F. 1999, ApJS, 120, 247

Gry, C., Swinyard, B., Harwood, A., et al. 2003, The ISO Handbook, Vol. III, LWS - The Long Wavelength Spectrometer, ESA SP-1262

Higdon, S. J. U., Higdon, J. L., van der Hulst, J. M., \& Stacey, G. J. 2003, ApJ, 592, 161

Howarth, I. D. 1983, MNRAS, 203, 301

Jamet, L., Pérez, E., Cerviño, M., et al. 2004, A\&A, 426, 399

Kennicutt, R. C. Jr., Bresolin, F., \& Garnett, D. R. 2003, ApJ, 591, 801

Leech, K., Kester, D., Shipman, R., et al. 2003, The ISO Handbook, Vol. V, SWS - The Short Wavelength Spectrometer, ESA SP-1262 Leitherer, C., Schaerer, D., Goldader, J. D., et al. 1999, ApJS, 123, 3 Lodders, K. 2003, ApJ, 591, 1220
Lee, M. G., Kim, M., Sarajedini, A., Geisler, D., \& Gieren, W. 2002, ApJ, 565, 959

Liu, X. W. 2003, IAUS, 209, 339

Luridiana, V., Peimbert, M., \& Leitherer, C. 1999, ApJ, 527, 110

Luridiana, V., Cerviño, M., \& Binette, L. 2001, A\&A, 379, 1017

Luridiana, V., \& Peimbert, M. 2001, ApJ, 553, 633

Luridiana, L., Peimbert, A., Peimbert, M., \& Cerviño, M. 2003, ApJ, 592,846

Maciejewski, W., Mathis, J. S., \& Edgar, R. J. 1996, ApJ, 462, 347

Maíz-Apellániz, J., Pérez, E., \& Mas-Hesse, J. M. 2004, AJ, 128, 1196

Mathis, J. S., Rumpl, W., \& Nordsieck, K. H. 1977, ApJ, 217, 425

Mohler, O. C. 1950, Photometric atlas of the near infra-red solar spectrum, $\lambda 8465$ to $\lambda 25,242$ (University of Michigan Press)

Moore, C. E., Minnaert, M. G. J., \& Houtgast, J. 1966, The Solar Spectrum $2935 \AA$ to $8770 \AA$, National Bureau of Standards

Nandy, K., Thompson, G. I., Jamar, C., Monfils, A., \& Wilson, R. 1975, A\&A, 44, 195

Osterbrock, D. E. 1974, Astrophysics of Gaseous Nebulae (W. H. Freeman \& co)

Péquignot, D., Petitjean, P., \& Boisson, C. 1991, A\&A, 251, 680

Peimbert, M. 1967, ApJ, 150, 825

Pietsch, W., Misanovic, Z., Haberl, F., et al. 2004, A\&A, 426, 11

Relaño, M., Peimbert, M., \& Beckman, J. 2002, ApJ, 564, 704

Seaton, M. J. 1979, MNRAS, 187, 73

Stasińska, G. 1990, A\&AS, 83, 501

Stasińska, G. 2004, in Cosmochemistry. The melting pot of the elements. XIII Canary Islands Winter School of Astrophysics, Puerto de la Cruz, Tenerife, Spain, November 19-30, 2001, ed. C. Esteban, R. J. García López, A. Herrero, \& F. Sánchez, Cambridge contemporary astrophysics, 115

Stasińska, G. 2005, A\&A, 434, 507

Stasińska, G., et al. 2005, in preparation

Stasińska, G., \& Schaerer, D. 1999, A\&A, 351, 72

Stasińska, G., \& Szczerba, R. 2001, A\&A, 379, 1024

Tenorio-Tagle, G. 1996, AJ, 111, 1641

Tsamis, Y. G., \& Péquignot, D. 2005, in preparation

Urbaneja, M. A., Herrero, A., Kudritski, P. P., et al., in preparation

Viallefond, F., Goss, W. M., van der Hulst, J. M., \& Crane, P. C. 1986, A\&AS, 64, 237

Vílchez, J. M., Pagel, B. E. J., Díaz, A. I., Terlevich, E., \& Edmunds, M. G. 1988, MNRAS, 235, 633

Walborn, N. R., Maíz-Apellániz, J., \& Barbá, R. H. 2002, AJ, 124, 1601 


\section{Online Material}




\section{Appendix A: Correction of Balmer lines used for dereddening}

Let us consider a set of hydrogen Balmer lines, whose fluxes are measured at a series of positions $x$ along a slit. At a given position $x$, the measured value of the flux of " $\mathrm{H} X$ ", $F_{X}(x)$, differs from its real value $F_{X}^{\text {real }}(x)$ by a measure error $\delta_{X}(x)=$ $F_{X}(x)-F_{X}^{\text {real }}(x)$. Furthermore, $F_{X}^{\text {real }}(x)$ is related to $F_{\beta}^{\text {real }}(x)$ by the following formula:

$$
\begin{aligned}
2.5 \log F_{X}^{\text {real }}(x)= & 2.5 \log \left(F_{\beta}^{\text {real }}(x) R_{X}\right) \\
& +E_{B-V}(x)\left(f_{\beta}-f_{X}\right)
\end{aligned}
$$

where $R_{X}$ is the theoretical $\mathrm{H} X / \mathrm{H} \beta$ intensity ratio, and $f_{x}$ the extinction law. By applying this equation to $\mathrm{H} \alpha$, we can derive the following formula, especially for the bright $\mathrm{H} \gamma$ and $\mathrm{H} \delta$ lines:

$$
\begin{aligned}
\left(f_{\beta}-f_{\alpha}\right) \ln \frac{F_{X}^{\mathrm{real}}(x)}{R_{X}}= & \left(f_{X}-f_{\alpha}\right) \ln F_{\beta}^{\mathrm{real}}(x) \\
& +\left(f_{\beta}-f_{X}\right) \ln \frac{F_{\alpha}^{\mathrm{real}}(x)}{R_{\alpha}}
\end{aligned}
$$

Analogously, we can write the flux of $\mathrm{H} X$ predicted from the observed fluxes of $\mathrm{H} \alpha$ and $\mathrm{H} \beta$, namely $F_{X}^{\text {pred }}(x)$. Due to the measure errors, $F_{X}^{\text {pred }}(x)$ and $F_{X}(x)$ are discrepant, and if the errors are small compared to the fluxes, then the discrepancy term $\Delta_{X}(x)=F_{X}(x)-F_{X}^{\text {pred }}(x)$ is given by:

$$
\begin{aligned}
\left(f_{\beta}-f_{\alpha}\right) \frac{\Delta_{X}(x)-\delta_{X}(x)}{F_{X}(x)}= & \left(f_{\alpha}-f_{X}\right) \frac{\delta_{\beta}(x)}{F_{\beta}(x)} \\
& +\left(f_{X}-f_{\beta}\right) \frac{\delta_{\alpha}(x)}{F_{\alpha}(x)} .
\end{aligned}
$$

We split the flux errors into three terms: spatially invariant residuals $\epsilon_{X}$ of the photometric calibration, stellar absorption features underlying the nebular Balmer lines, and the usual line flux dispersions (such as photon noise) $\xi_{X}(x)=0 \pm \sigma_{X}(x)$. The equivalent widths of the stellar lines are approximately the same for $\mathrm{H} \alpha, \mathrm{H} \beta$, and $\mathrm{H} \gamma$ (e.g., Auer \& Mihalas 1972), but may vary spatially if the stellar content is heterogeneous. Consequently, we described the stellar contribution to the flux errors as the sum of the contributions of different subsets $i$ of the cluster, each being associated to an equivalent width $W_{i}$ and continua $F_{\lambda X}^{i}(x)$. The sum of the errors $\delta_{X}(x)$ is:

$$
\frac{\delta_{X}(x)}{F_{X}(x)}=\epsilon_{X}-\sum_{i} W_{i} \frac{F_{\lambda X}^{i}(x)}{F_{X}(x)} \pm \frac{\sigma_{X}(x)}{F_{X}(x)}
$$

The linear Eq. (A.3) applies if the relative errors $\delta_{X}(x) / F_{X}(x)$ are small, i.e. if the photometric errors $\epsilon_{X}$ are much smaller than 1 , if the equivalent widths of the nebular lines with respect to the stellar continuum are much greater than the ones of the stellar lines, and if the signal-to-noise ratios $(\mathrm{S} / \mathrm{N}) F_{X}(x) / \sigma_{X}(x)$ are high.

\section{A.1. Photometric errors}

Let us consider the set of positions $x$ unaffected by stellar absorption lines, and where the nebular lines were measured with high S/N. Combining Eqs. (A.3) and (A.4) give:

$$
\begin{aligned}
\left(f_{\beta}-f_{\alpha}\right) \frac{\Delta_{X}(x)}{F_{X}(x)}= & \left(f_{X}-f_{\beta}\right) \epsilon_{\alpha}+\left(f_{\alpha}-f_{X}\right) \epsilon_{\beta} \\
& +\left(f_{\beta}-f_{\alpha}\right) \epsilon_{X} \pm \tau_{X}(x)
\end{aligned}
$$

with

$$
\begin{aligned}
\tau_{X}^{2}(x)= & \left(f_{X}-f_{\beta}\right)^{2}\left(\frac{\sigma_{\alpha}(x)}{F_{\alpha}(x)}\right)^{2}+\left(f_{\alpha}-f_{X}\right)^{2}\left(\frac{\sigma_{\beta}(x)}{F_{\beta}(x)}\right)^{2} \\
& +\left(f_{\beta}-f_{\alpha}\right)^{2}\left(\frac{\sigma_{X}(x)}{F_{X}(x)}\right)^{2} .
\end{aligned}
$$

The contribution $\eta_{X}$ of the photometric residuals to $\Delta_{X}(x) / F_{X}(x)$ can be estimated with the following formula:

$\eta_{X}=\frac{\sum_{x}\left(\Delta_{X}(x) / F_{X}(x)\right) / \tau_{X}^{2}(x)}{\sum_{x} 1 / \tau_{X}^{2}(x)}$

$\eta_{X}$ relates $\epsilon_{X}$ to $\epsilon_{\alpha}$ and $\epsilon_{\beta}$ :

$\epsilon_{X}=\eta_{X}-\frac{f_{X}-f_{\beta}}{f_{\beta}-f_{\alpha}} \epsilon_{\alpha}-\frac{f_{\alpha}-f_{X}}{f_{\beta}-f_{\alpha}} \epsilon_{\beta}$.

Since they are residuals of the fit of the instrumental photometric response, we can write $\epsilon_{X}=0 \pm \phi_{X}$. Furthermore, if they are not correlated between them, we can assume they minimize the error balance $\chi^{2}=\sum_{X} \epsilon_{X}^{2} / \phi_{X}^{2}$, more specifically given by:

$\chi^{2}\left(\epsilon_{\alpha}, \epsilon_{\beta}\right)=\sum_{X} \frac{1}{\phi_{X}^{2}}\left(\frac{f_{X}-f_{\beta}}{f_{\beta}-f_{\alpha}} \epsilon_{\alpha}+\frac{f_{\alpha}-f_{X}}{f_{\beta}-f_{\alpha}} \epsilon_{\beta}-\eta_{X}\right)^{2}$.

The estimations and uncertainties of $\epsilon_{\alpha}, \epsilon_{\beta}$, and more generally $\epsilon_{X}$ can be easily derived from this linear $\chi^{2}$ and from Eq. (A.8). The correction of the line fluxes for these errors consists in multiplying them by $1-\epsilon_{X}$.

\section{A.2. Underlying stellar absorption lines}

Making use of fluxes corrected for photometric errors and measured with high signal-no-noise ratio, we can write, for $\mathrm{H} \gamma$ :

$\left(f_{\beta}-f_{\alpha}\right) \frac{\Delta_{\gamma}(x)}{F_{\gamma}(x)}=-\sum_{i} W_{i} \omega_{i}(x) \pm \tau_{\gamma}(x)$

$$
\begin{aligned}
\omega_{i}(x)= & \left(f_{\gamma}-g_{\beta}\right) \frac{F_{\lambda \alpha}^{i}(x)}{F_{\alpha}(x)}+\left(f_{\alpha}-f_{\gamma}\right) \frac{F_{\lambda \beta}^{i}(x)}{F_{\beta}(x)} \\
& +\left(f_{\beta}-f_{\alpha}\right) \frac{F_{\lambda \gamma}^{i}(x)}{F_{\gamma}(x)} .
\end{aligned}
$$

The estimate of the equivalent widths $W_{i}$ is given by the minimization of the following linear $\chi^{2}$ :

$$
\chi^{2}=\sum_{x} \frac{1}{\tau_{\gamma}^{2}(x)}\left(\sum_{i} W_{i} \omega_{i}(x)+\left(f_{\beta}-f_{\alpha}\right) \frac{\Delta_{\gamma}(x)}{F_{\gamma}(x)}\right)^{2} .
$$


L. Jamet et al.: The giant HII region NGC 588, Online Material $p 3$

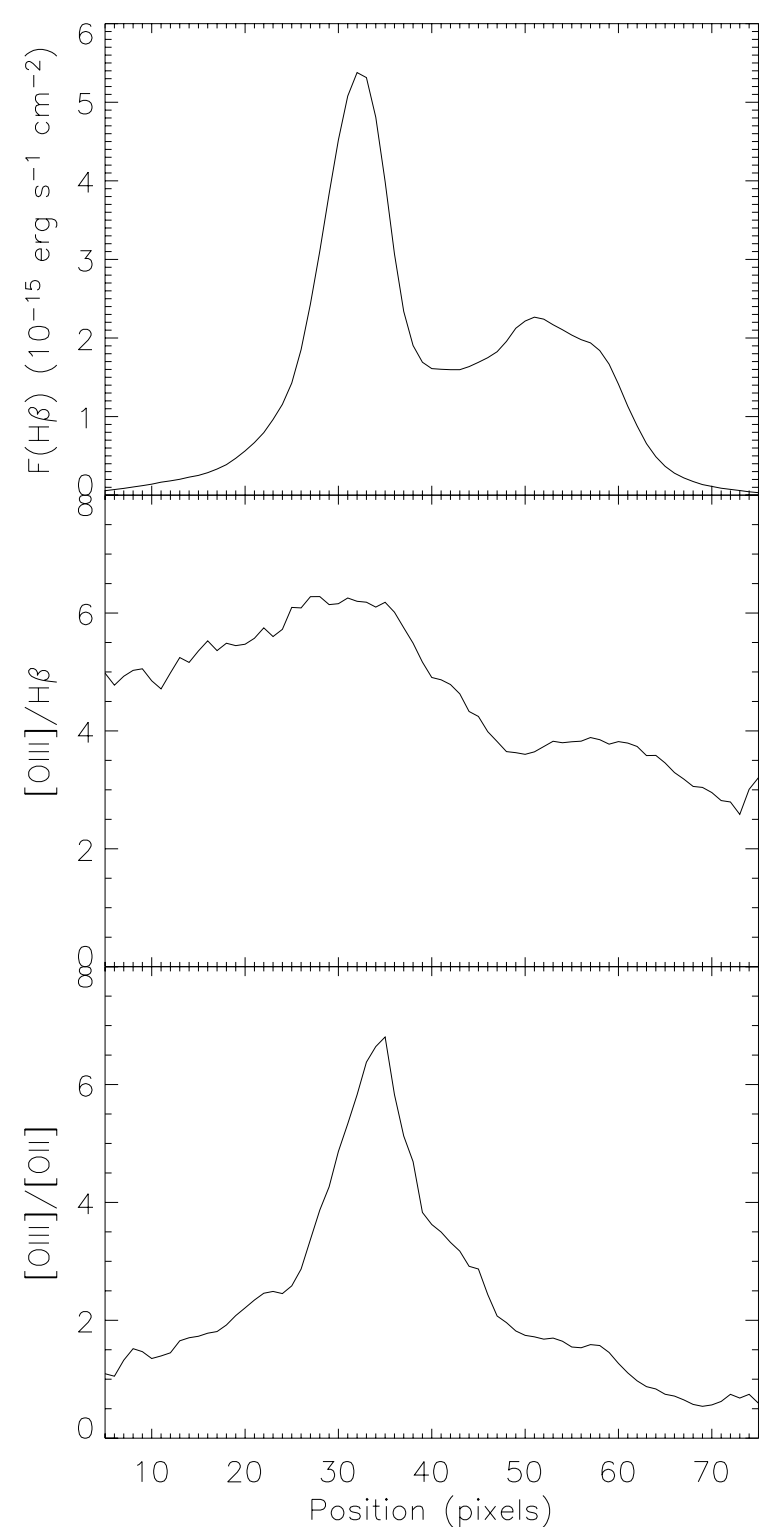

Fig. B.1. Profiles of $\mathrm{H} \beta$ (upper panel), [OIII] $\lambda 5007 / \mathrm{H} \beta$ (middle panel), and [OIII] $\lambda 5007 /[\mathrm{OII}] \lambda 372(6+9)$ (lower panel) along the optical slit.

\section{Appendix B: Setting the geometric corrections $A$, $B$, and $K$}

We used the well-observed long-slit profiles of $\mathrm{H} \beta$, [OIII] $\lambda 5007$, and [OII] $\lambda 372(6+9)$, and the [OIII] $\lambda 5007 / \mathrm{H} \alpha$ map to constrain the coefficients $A, B$ and $K$. From those data (Figs. B.1 and B.2), it is evident that the $\operatorname{knot} G$ is more excited than $S$, even though its rms density is higher than the latter. This can be easily explained by its short distance to the stellar source (Sect. 6.1.1).
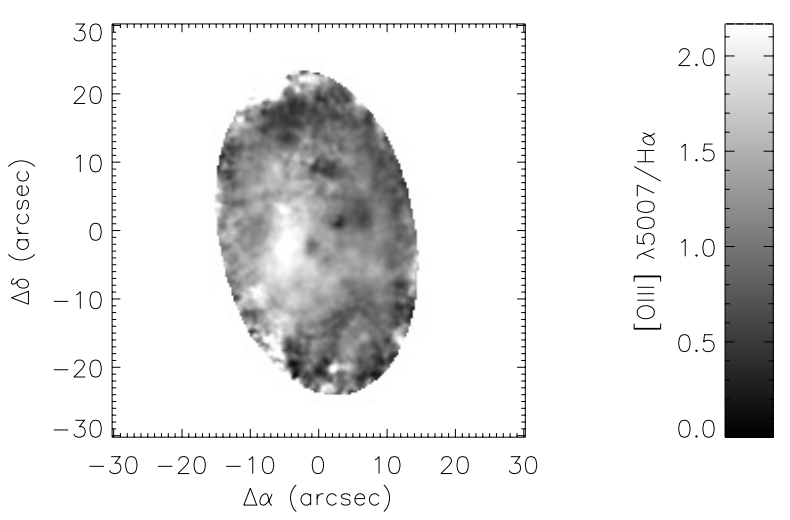

Fig. B.2. Map of the nebular [OIII] $\lambda 5007 / \mathrm{H} \alpha$ ratio. The ratio is too noisy to be shown outside the ellipse cutout.

Coefficients $A$ and $B$ strongly influence the predicted ionization state of $S$, while $K$ acts on the ionization state of $G$. We decided to set them, for each model, with the following method.

We manually separated the contributions of $S$ and $G$ to the long-slit fluxes of [OIII] $\lambda 5007$ and [OII] $\lambda 372(6+9)$, and derived the values of $[\mathrm{OIII}] \lambda 5007 /[\mathrm{OII}] \lambda 372(6+9)$ for both components. We found $F([\mathrm{OIII}]) / F([\mathrm{OII}])=2.14 \pm 0.15$ for $S$ and $5.68 \pm 0.65$ for $G$.

For each model, we first computed the predicted fluxes of the lines observed through the optical slit. Unless otherwise mentioned, we set the coefficients $A$ and $K$ to reproduce the [OIII] $\lambda 5007 /[\mathrm{OII}] \lambda 372(6+9)$ ratios of $S$ and $G$. Once those coefficients were set, we reported the ratio $R$ between the predicted and the observed value of [OIII] $\lambda 5007 / \mathrm{H} \beta$. Then, we calculated the model line fluxes integrated over the whole nebula. In the latter calculation, coefficient $B$ was chosen so as to reproduce the model/observation ratio $R$, but for the integrated lines this time.

The geometric transforms performed on the gas density distributions $(f(r)$ for $S, g(r)$ for $G$ ) were accompanied with a renormalization of the latter to conserve the predicted $\mathrm{H} \beta$ fluxes. Indeed, the integrated volume of gas in $S$ is proportional to $A^{2}$ for the optical slit and to $B^{3}$ for the whole nebula, while the volume of $G$ is proportional to $K$. Since the local $\mathrm{H} \beta$ emissivity is proportional to the square density, the transform of $f(r)$ consisted in replacing it by $f(r / A) / A$ (optical slit) or $f(r / B) / B^{3 / 2}$ (whole volume), and $g(r)$ was replaced by $g(r) / K^{1 / 2}$. 